CERN-TH.6150/91

ETH-TH/91-7

\title{
Testing the Higgs Sector of the Minimal Supersymmetric Standard Model at Large Hadron Colliders
}

\author{
Z. Kunszt \\ Institute of Theoretical Physics, ETH, \\ Zurich, Switzerland \\ and \\ F. Zwirner円 \\ Theory Division, CERN, \\ Geneva, Switzerland
}

\begin{abstract}
We study the Higgs sector of the Minimal Supersymmetric Standard Model, in the context of proton-proton collisions at LHC and SSC energies. We assume a relatively heavy supersymmetric particle spectrum, and include recent results on one-loop radiative corrections to Higgs-boson masses and couplings. We begin by discussing present and future constraints from the LEP experiments. We then compute branching ratios and total widths for the neutral $(h, H, A)$ and charged $\left(H^{ \pm}\right)$Higgs particles. We present total cross-sections and event rates for the important discovery channels at the LHC and SSC. Promising
\end{abstract}

\footnotetext{
${ }^{1}$ On leave from INFN, Sezione di Padova, Italy.
} 
physics signatures are given by $h \rightarrow \gamma \gamma, H \rightarrow \gamma \gamma$ or $Z^{*} Z^{*}$ or $\tau^{+} \tau^{-}$, $A \rightarrow \tau^{+} \tau^{-}$, and $t \rightarrow b H^{+}$followed by $H^{+} \rightarrow \tau^{+} \nu_{\tau}$, which should allow for an almost complete coverage of the parameter space of the model.

CERN-TH.6150/91

ETH-TH/91-7

December 1991 


\section{Introduction}

All available experimental data in particle physics are consistent with the Standard Model (SM) of strong and electroweak interactions, provided [1]

$$
91 \mathrm{GeV}<m_{t}<180 \mathrm{GeV} \quad(95 \% \text { c.l. })
$$

and

$$
57 \mathrm{GeV}<m_{\varphi} \quad(95 \% \text { c.l. }),
$$

where $m_{t}$ and $m_{\varphi}$ denote the masses of the top quark and of the SM Higgs boson, respectively. The lower limits on $m_{t}$ and $m_{\varphi}$ are obtained from unsuccessful direct searches at the Tevatron and LEP. The upper limit on $m_{t}$ is obtained as a consistency condition of the SM, after the inclusion of radiative corrections, with the high-precision data on electroweak phenomena. Strong evidence for the existence of the top quark, with the quantum numbers predicted by the SM, is also provided by the precise measurements of the weak isospin of the $b$-quark. In the case of the Higgs boson, the situation is radically different. There is no experimental evidence yet that the minimal SM Higgs mechanism is the correct description of electroweak symmetry breaking. Fortunately, present and future accelerators will give decisive contributions towards the experimental solution of this problem. If the SM description of the Higgs mechanism is correct, LEP or the LHC and SSC should be able to find the SM Higgs boson and study its properties.

Despite its remarkable successes, the SM can only be regarded as an effective low-energy theory, valid up to some energy scale $\Lambda$ at which it is replaced by some more fundamental theory. Certainly $\Lambda$ is less than the Planck scale, $M_{\mathrm{P}} \sim 10^{19} \mathrm{GeV}$, since one needs a theory of quantum gravity to describe physics at these energies. However, there are also arguments, originating precisely from the study of the untested Higgs sector \&, which suggest that $\Lambda$ should rather be close to the Fermi scale $G_{\mathrm{F}}^{-1 / 2} \sim 300 \mathrm{GeV}$. The essence of these arguments is the following. Triviality of the $\lambda \varphi^{4}$ theory, absence of Landau poles and perturbative unitarity imply that within the SM $m_{\varphi}<600-800 \mathrm{GeV}$. If one then tries to extend the validity of the SM to energy scales $\Lambda \gg G_{\mathrm{F}}^{-1 / 2}$, one is faced with the fact that in the SM there is no symmetry to justify the smallness of the Higgs mass with respect to

\footnotetext{
${ }^{2}$ For reviews of Higgs boson physics see, e.g., refs. [2,3]
} 
the (physical) cut-off $\Lambda$. This is apparent from the fact that in the SM oneloop radiative corrections to the Higgs mass are quadratically divergent; it is known as the naturalness (or hierarchy) problem of the SM. Motivated by this problem, much theoretical effort has been devoted to finding descriptions of electroweak symmetry breaking which modify the SM at scales $\Lambda \sim G_{\mathrm{F}}^{-1 / 2}$. The likely possibility of such modifications is the reason why, when discussing the experimental study of electroweak symmetry breaking, one should not be confined to the SM Higgs, but also consider alternatives to it, which might have radically different signatures, and in some cases be more difficult to detect than the SM Higgs. Only after a thorough study of these alternatives can one be definite about the validity of the so-called 'no-lose theorems', stating that the physics signatures of electroweak symmetry breaking cannot be missed at LEP or the LHC and SSC.

When considering alternatives to the minimal SM Higgs sector, it is natural to concentrate on models which are theoretically motivated, phenomenologically acceptable and calculationally well-defined. The most attractive possibility satisfying these criteria is the Minimal Supersymmetric Standard Model (MSSM) [4]. This possibility is theoretically motivated by the fact that low-energy supersymmetry, effectively broken in the vicinity of the electroweak scale, is the only theoretical framework that can naturally accommodate elementary Higgs bosons. The simplest and most predictive realization of low-energy supersymmetry is the MSSM, defined by 1) minimal gauge group: $\left.S U(3)_{C} \times S U(2)_{L} \times U(1)_{Y} ; 2\right)$ minimal particle content: three generations of quarks and leptons and two Higgs doublets, plus their superpartners; $3)$ an exact discrete $R$-parity, which guarantees (perturbative) baryon- and lepton-number conservation: $R=+1$ for SM particles and Higgs bosons, $R=-1$ for their superpartners; 4) supersymmetry breaking parametrized by explicit but soft breaking terms: gaugino and scalar masses and trilinear scalar couplings.

Besides the solution of the naturalness problem, there are other virtues of the MSSM which are not shared by many other alternatives to the SM Higgs and should also be recalled to further motivate our study. The MSSM successfully survives all the stringent phenomenological tests coming from precision measurements at LEP: in most of its parameter space, the MSSM predictions for the LEP observables are extremely close to the SM predictions, evaluated for a relatively light SM Higgs [5]. This can be compared, for example, with the simplest technicolor models, which are ruled out by the re- 
cent LEP data [6]. Again in contrast with models of dynamical electroweak symmetry breaking, the MSSM has a high degree of predictivity, since all masses and couplings of the Higgs boson sector can be computed, at the tree-level, in terms of only two parameters, and radiative corrections can be kept under control: in particular, cross-sections and branching ratios for the MSSM Higgs bosons can be reliably computed in perturbation theory. Furthermore, it is intriguing that the idea of grand unification, which fails in its minimal non-supersymmetric implementation, can be successfully combined with that of low-energy supersymmetry: minimal supersymmetric grand unification predicts a value of $\sin ^{2} \theta_{W}\left(m_{Z}\right)$ which is in good agreement with the measured one, and a value of the grand-unification mass which could explain why proton decay has escaped detection so far [7]. Finally, as a consequence of $R$-parity, the lightest supersymmetric particle, which is typically neutral and weakly interacting, is absolutely stable, and thus a natural candidate for dark matter.

Any consistent supersymmetric extension of the SM requires at least two Higgs doublets, in order to give masses to all charged quarks and leptons and to avoid gauge anomalies originated by the spin- $1 / 2$ higgsinos. The MSSM has just two complex Higgs doublets, with the following $S U(3)_{C} \times S U(2)_{L} \times$ $U(1)_{Y}$ quantum numbers $\left(Q=T_{3 L}+Y\right)$ :

$$
H_{1} \equiv\left(\begin{array}{c}
H_{1}^{0} \\
H_{1}^{-}
\end{array}\right) \sim(1,2,-1 / 2), \quad H_{2} \equiv\left(\begin{array}{c}
H_{2}^{+} \\
H_{2}^{0}
\end{array}\right) \sim(1,2,+1 / 2) .
$$

Other non-minimal models can be constructed, but they typically increase the number of parameters without correspondingly increasing the physical motivation. For example, the simplest non-minimal model [8] is constructed by adding a singlet Higgs field $N$ and by requiring purely trilinear superpotential couplings. In this model, the Higgs sector has already two more parameters than in the MSSM. Folklore arguments in favour of this model are that it avoids the introduction of a supersymmetry-preserving mass parameter $\mu \sim G_{\mathrm{F}}^{-1 / 2}$ and that the homogeneity properties of its superpotential recall the structure of some superstring effective theories. A closer look, however, shows that these statements should be taken with a grain of salt. First, in the low-energy effective theory with softly broken global supersymmetry, the supersymmetric mass $\mu \sim G_{\mathrm{F}}^{-1 / 2}$ could well be a remnant of local supersymmetry breaking, if the underlying supergravity theory has a suitable structure of interactions [9]. Moreover, when embedded in a grand-unified theory, the 
non-minimal model with a singlet Higgs field might develop dangerous instabilities [10]. Also, the trilinear $N^{3}$ superpotential coupling, which is usually invoked to avoid a massless axion, is typically absent in string models. We therefore concentrate in this paper on the MSSM only.

The previous considerations should have convinced the reader that the Higgs sector of the MSSM is worth a systematic study in view of the forthcoming hadron colliders, the LHC and SSC. To perform such a study, one has to deal with the rich particle spectrum of the MSSM. As discussed in more detail later, the Higgs sector contains one charged $\left(H^{ \pm}\right)$and three neutral $(h, H, A)$ physical states. At the classical level, all Higgs boson masses and couplings can be expressed in terms of two parameters only, for example $m_{A}$ and $\tan \beta \equiv v_{2} / v_{1}$. This makes the discussion more complicated than in the SM, where the only free parameter in the Higgs sector is the Higgs mass, $m_{\varphi}$. In addition, when considering production and decay of Higgs bosons, the whole particle spectrum of the model has to be taken into account. As in the SM, the top-quark mass $m_{t}$ is an important parameter: barring the fine-tuned cases of a very light stop squark, or of charginos very close in mass to $m_{Z} / 2$, the limits of eq. (1) are also valid in the MSSM [5]. In contrast with the SM, also the supersymmetric $R$-odd particles (squarks, sleptons, gauginos, higgsinos) can play an important role in the production and decay of supersymmetric Higgs bosons [11]. Clearly, to keep track simultaneously of all supersymmetric-particle masses would be a difficult (and confusing) task. We shall therefore concentrate, following the approach of ref. [12], on the limiting case where all supersymmetric-particle masses are heavy enough not to play an important role in the phenomenology of supersymmetric Higgs bosons. This is phenomenologically meaningful, since one can argue that a relatively light supersymmetric-particle spectrum is likely to give independent, detectable signatures at LEP or at the LHC and SSC.

Another motivation for the present study is the recent realization [13] that tree-level formulae for Higgs-boson masses and couplings can receive large radiative corrections, dominated by the exchange of virtual top and bottom quarks and squarks in loop diagrams. For example, tree-level formulae would predict the existence of a neutral Higgs boson $(h)$ lighter than the $Z$. If this were true, there would be a chance of testing completely the MSSM Higgs sector at LEP II, with no need for the LHC and SSC. However, $m_{h}$ can receive a large positive shift by radiative corrections, which can push $h$ beyond the LEP II discovery reach. This makes the LHC and SSC important, not only 
for a possible confirmation of a SUSY Higgs signal seen at LEP, but also for the exploration of the parameter space inaccessible to LEP.

The phenomenology of the SM Higgs at the LHC [14 [16] and SSC [17, 18] has been intensely studied over the last years: a lot of effort was required to prove [14, 15], at least on paper, that the combination of LEP and the LHC/SSC is sufficient to explore the full theoretically allowed range of SM Higgs masses. However, those results cannot be directly applied to the neutral states of the MSSM, since there are important differences in the couplings, and of course one needs to analyse separately the case of the charged Higgs. Even in the case in which all the $R$-odd supersymmetric particles are very heavy, the Higgs sector of the MSSM represents a non-trivial extension of the SM case. Also several studies of the MSSM Higgs sector have already appeared in the literature. In particular, tree-level formulae for the MSSM Higgs boson masses and couplings are available, and they have already been used to compute cross-sections and branching ratios for representative values of the MSSM parameters [3]. However, the existing analyses are not systematic enough to allow for a definite conclusion concerning the discovery potential of the LHC and SSC, even in the simple case of large sparticle masses. Also, they do not include radiative corrections to Higgs-boson masses and couplings. In this paper we plan to help filling these two gaps. The strategy for a systematic study of neutral supersymmetric Higgs bosons at the LHC was outlined in ref. [12]: however, at that time radiative corrections were not available, and also the $\gamma \gamma$ branching ratio was incorrectly encoded in the computer program. Our goal will be to see if LEP and the LHC/SSC can be sensitive to supersymmetric Higgs bosons in the whole $\left(m_{A}, \tan \beta\right)$ space.

The structure of the paper is the following. In sect. 2 we review the theoretical structure of the Higgs sector of the MSSM, including radiatively corrected formulae for Higgs-boson masses and couplings. In sect. 3 we survey the present LEP I limits, after the inclusion of radiative corrections, and the plausible sensitivity of LEP II. In sect. 4 we present branching ratios and widths of neutral and charged supersymmetric Higgs bosons. In sect. 5 we compute the relevant cross-sections at the LHC and SSC, and in sect. 6 we examine in some detail the most promising signals for discovery. Finally, sect. 7 contains a concluding discussion of our results and of prospects for further work. 


\section{Higgs masses and couplings in the MSSM}

For a discussion of Higgs-boson masses and couplings in the MSSM, the obvious starting point is the tree-level Higgs potential [4]

$$
\begin{aligned}
V_{0}= & m_{1}^{2}\left|H_{1}\right|^{2}+m_{2}^{2}\left|H_{2}\right|^{2}+m_{3}^{2}\left(H_{1} H_{2}+\text { h.c. }\right) \\
& +\frac{1}{8} g^{2}\left(H_{2}^{\dagger} \vec{\sigma} H_{2}+H_{1}^{\dagger} \vec{\sigma} H_{1}\right)^{2}+\frac{1}{8} g^{\prime 2}\left(\left|H_{2}\right|^{2}-\left|H_{1}\right|^{2}\right)^{2},
\end{aligned}
$$

where $m_{1}^{2}, m_{2}^{2}, m_{3}^{2}$ are essentially arbitrary mass parameters, $g$ and $g^{\prime}$ are the $S U(2)$ and $U(1)$ coupling constants, respectively, and $\vec{\sigma}$ are the Pauli matrices. $S U(2)$ indices are left implicit and contracted in the obvious way. It is not restrictive to choose $m_{3}^{2}$ real and negative, and then the vacuum expectation values $v_{1} \equiv\left\langle H_{1}^{0}\right\rangle$ and $v_{2} \equiv\left\langle H_{2}^{0}\right\rangle$ real and positive.

The physical states of the MSSM Higgs sector are three neutral bosons (two CP-even, $h$ and $H$, and one CP-odd, $A$ ) and a charged boson, $H^{ \pm}$. A physical constraint comes from the fact that the combination $\left(v_{1}^{2}+v_{2}^{2}\right)$, which determines the $W$ and $Z$ boson masses, must reproduce their measured values. Once this constraint is imposed, in the Born approximation the MSSM Higgs sector contains only two independent parameters. A convenient choice, which will be adopted throughout this paper, is to take as independent parameters $m_{A}$, the physical mass of the CP-odd neutral boson, and $\tan \beta \equiv v_{2} / v_{1}$, where $v_{1}$ gives mass to charged leptons and quarks of charge $-1 / 3, v_{2}$ gives mass to quarks of charge $2 / 3$. The parameter $m_{A}$ is essentially unconstrained, even if naturalness arguments suggest that it should be smaller than $O(500 \mathrm{GeV})$, whereas for $\tan \beta$ the range permitted by model calculations is $1 \leq \tan \beta \lesssim \frac{m_{t}}{m_{b}}$.

At the classical level, the mass matrix of neutral CP-even Higgs bosons reads

$$
\left(\mathcal{M}_{R}^{0}\right)^{2}=\left[\left(\begin{array}{cc}
\cot \beta & -1 \\
-1 & \tan \beta
\end{array}\right) \frac{m_{Z}^{2}}{2}+\left(\begin{array}{cc}
\tan \beta & -1 \\
-1 & \cot \beta
\end{array}\right) \frac{m_{A}^{2}}{2}\right] \sin 2 \beta
$$

and the charged-Higgs mass is given by

$$
m_{H^{ \pm}}^{2}=m_{W}^{2}+m_{A}^{2}
$$

From eq. (5), one obtains

$$
m_{h, H}^{2}=\frac{1}{2}\left[m_{A}^{2}+m_{Z}^{2} \mp \sqrt{\left(m_{A}^{2}+m_{Z}^{2}\right)^{2}-4 m_{A}^{2} m_{Z}^{2} \cos ^{2} 2 \beta}\right],
$$




\begin{tabular}{|c|c|c|c|}
\hline & $\begin{array}{c}d \bar{d}, s \bar{s}, b \bar{b} \\
e^{+} e^{-}, \mu^{+} \mu^{-}, \tau^{+} \tau^{-}\end{array}$ & $u \bar{u}, c \bar{c}, t \bar{t}$ & $W^{+} W^{-}, Z Z$ \\
\hline$h$ & $-\sin \alpha / \cos \beta$ & $\cos \alpha / \sin \beta$ & $\sin (\beta-\alpha)$ \\
\hline$H$ & $\cos \alpha / \cos \beta$ & $\sin \alpha / \sin \beta$ & $\cos (\beta-\alpha)$ \\
\hline$A$ & $-i \gamma_{5} \tan \beta$ & $-i \gamma_{5} \cot \beta$ & 0 \\
\hline
\end{tabular}

Table 1: Correction factors for the couplings of the MSSM neutral Higgs bosons to fermion and vector boson pairs.

and also celebrated inequalities such as $m_{W}, m_{A}<m_{H^{ \pm}}, m_{h}<m_{Z}<m_{H}$, $m_{h}<m_{A}<m_{H}$. Similarly, one can easily compute all the Higgs-boson couplings by observing that the mixing angle $\alpha$, required to diagonalize the mass matrix (5), is given by

$$
\cos 2 \alpha=-\cos 2 \beta \frac{m_{A}^{2}-m_{Z}^{2}}{m_{H}^{2}-m_{h}^{2}}, \quad-\frac{\pi}{2}<\alpha \leq 0 .
$$

For example, the couplings of the three neutral Higgs bosons are easily obtained from the SM Higgs couplings if one multiplies them by the $\alpha$ - and $\beta$-dependent factors summarized in table 1. The remaining tree-level Higgsboson couplings in the MSSM can be easily computed and are summarized, for example, in ref. [3]. An important consequence of the structure of the classical Higgs potential of eq. (4) is the existence of at least one neutral CP-even Higgs boson, weighing less than or about $m_{Z}$ and with approximately standard couplings to the $Z$. This raised the hope that the crucial experiment on the MSSM Higgs sector could be entirely performed at LEP II (with sufficient centre-of-mass energy, luminosity and b-tagging efficiency), and took some interest away from the large hadron collider environment. 
However, it was recently pointed out [13] that the masses of the Higgs bosons in the MSSM are subject to large radiative corrections, associated with the top quark and its $S U(2)$ and supersymmetric partners ${ }^{\text {f }}$. Several papers [20 23] have subsequently investigated various aspects of these corrections and their implications for experimental searches at LEP. In the rest of this section, we shall summarize and illustrate the main effects of radiative corrections on Higgs-boson parameters.

As far as Higgs-boson masses and self-couplings are concerned, a convenient approximate way of parametrizing one-loop radiative corrections is to substitute the tree-level Higgs potential of eq. (4) with the one-loop effective potential, and to identify Higgs-boson masses and self-couplings with the appropriate combinations of derivatives of the effective potential, evaluated at the minimum. The comparison with explicit diagrammatic calculations shows that the effective potential approximation is more than adequate for our purposes. Also, inspection shows that the most important corrections are due to loops of top and bottom quarks and squarks. At the minimum $\left\langle H_{1}^{0}\right\rangle=v_{1},\left\langle H_{2}^{0}\right\rangle=v_{2},\left\langle H_{1}^{-}\right\rangle=\left\langle H_{2}^{+}\right\rangle=0$, and neglecting intergenerational mixing, one obtains for the top and bottom quark and squark masses the familiar expressions

$$
\begin{aligned}
& m_{t}^{2}=h_{t}^{2} v_{2}^{2}, \quad m_{b}^{2}=h_{b}^{2} v_{1}^{2}, \\
& m_{\tilde{t}_{1,2}}^{2}=m_{t}^{2}+\frac{1}{2}\left(m_{Q}^{2}+m_{U}^{2}\right)+\frac{1}{4} m_{Z}^{2} \cos 2 \beta \\
& \pm \sqrt{\left[\frac{1}{2}\left(m_{Q}^{2}-m_{U}^{2}\right)+\frac{1}{12}\left(8 m_{W}^{2}-5 m_{Z}^{2}\right) \cos 2 \beta\right]^{2}+m_{t}^{2}\left(A_{t}+\mu \cot \beta\right)^{2}}, \\
& m_{\tilde{b}_{1,2}}^{2}=m_{b}^{2}+\frac{1}{2}\left(m_{Q}^{2}+m_{D}^{2}\right)-\frac{1}{4} m_{Z}^{2} \cos 2 \beta \\
& \pm \sqrt{\left[\frac{1}{2}\left(m_{Q}^{2}-m_{D}^{2}\right)-\frac{1}{12}\left(4 m_{W}^{2}-m_{Z}^{2}\right) \cos 2 \beta\right]^{2}+m_{b}^{2}\left(A_{b}+\mu \tan \beta\right)^{2}} \text {. }
\end{aligned}
$$

\footnotetext{
${ }^{3}$ Previous studies [19] either neglected the case of a heavy top quark, or concentrated on the violations of the neutral-Higgs mass sum rule without computing corrections to individual Higgs masses.
} 
In eqs. (9) to (11), $h_{t}$ and $h_{b}$ are the top and bottom Yukawa couplings, and $m_{Q}, m_{U}, m_{D}$ are soft supersymmetry-breaking squark masses. The parameters $A_{t}, A_{b}$ and $\mu$, which determine the amount of mixing in the stop and sbottom mass matrices, are defined by the trilinear potential terms $h_{t} A_{t}\left(\tilde{t}_{L} \tilde{t}^{c}{ }_{L} H_{2}^{0}-\tilde{b}_{L} \tilde{t}^{c}{ }_{L} H_{2}^{+}\right)+$h.c., $h_{b} A_{b}\left(\tilde{b}_{L} \tilde{b}^{c}{ }_{L} H_{1}^{0}-\tilde{t}_{L} \tilde{b}^{c}{ }_{L} H_{1}^{-}\right)+$h.c. and by the superpotential mass term $\mu\left(H_{1}^{0} H_{2}^{0}-H_{1}^{-} H_{2}^{+}\right)$, respectively.

To simplify the discussion, in the following we will take a universal soft supersymmetry-breaking squark mass,

$$
m_{Q}^{2}=m_{U}^{2}=m_{D}^{2} \equiv m_{\tilde{q}}^{2}
$$

and we will assume negligible mixing in the stop and sbottom mass matrices,

$$
A_{t}=A_{b}=\mu=0
$$

Formulae valid for arbitrary values of the parameters can be found in refs. [22,23], but the qualitative features corresponding to the parameter choice of eqs. (12) and (13) are representative of a very large region of parameter space. In the case under consideration, and neglecting $D$-term contributions to the field-dependent stop and sbottom masses, the neutral CP-even mass matrix is modified at one loop as follows

$$
\mathcal{M}_{R}^{2}=\left(\mathcal{M}_{R}^{0}\right)^{2}+\left(\begin{array}{cc}
\Delta_{1}^{2} & 0 \\
0 & \Delta_{2}^{2}
\end{array}\right)
$$

where

$$
\begin{aligned}
\Delta_{1}^{2} & =\frac{3 g^{2} m_{b}^{4}}{16 \pi^{2} m_{W}^{2} \cos ^{2} \beta} \log \frac{m_{\tilde{b}_{1}}^{2} m_{\tilde{b}_{2}}^{2}}{m_{b}^{4}}, \\
\Delta_{2}^{2} & =\frac{3 g^{2} m_{t}^{4}}{16 \pi^{2} m_{W}^{2} \sin ^{2} \beta} \log \frac{m_{\tilde{t}_{1}}^{2} m_{\tilde{t}_{2}}^{2}}{m_{t}^{4}} .
\end{aligned}
$$

From the above expressions one can easily derive the one-loop-corrected eigenvalues $m_{h}$ and $m_{H}$, as well as the mixing angle $\alpha$ associated with the one-loop-corrected mass matrix (14). The one-loop-corrected charged Higgs mass is given instead by

$$
m_{H^{ \pm}}^{2}=m_{W}^{2}+m_{A}^{2}+\Delta^{2}
$$


where, including $D$-term contributions to stop and sbottom masses,

$$
\begin{aligned}
\Delta^{2}= & \frac{3 g^{2}}{64 \pi^{2} \sin ^{2} \beta \cos ^{2} \beta m_{W}^{2}} \\
& \times\left\{\frac{\left(m_{b}^{2}-m_{W}^{2} \cos ^{2} \beta\right)\left(m_{t}^{2}-m_{W}^{2} \sin ^{2} \beta\right)}{m_{\tilde{t}_{1}}^{2}-m_{\tilde{b}_{1}}^{2}}\left[f\left(m_{\tilde{t}_{1}}^{2}\right)-f\left(m_{\tilde{b}_{1}}^{2}\right)\right]\right. \\
& \left.+\frac{m_{t}^{2} m_{b}^{2}}{m_{\tilde{t}_{2}}^{2}-m_{\tilde{b}_{2}}^{2}}\left[f\left(m_{\tilde{t}_{2}}^{2}\right)-f\left(m_{\tilde{b}_{2}}^{2}\right)\right]-\frac{2 m_{t}^{2} m_{b}^{2}}{m_{t}^{2}-m_{b}^{2}}\left[f\left(m_{t}^{2}\right)-f\left(m_{b}^{2}\right)\right]\right\} \text { (18) }
\end{aligned}
$$

and

$$
f\left(m^{2}\right)=2 m^{2}\left(\log \frac{m^{2}}{Q^{2}}-1\right) .
$$

The most striking fact in eqs. (14)-(19) is that the correction $\Delta_{2}^{2}$ is proportional to $\left(m_{t}^{4} / m_{W}^{2}\right)$. This implies that, for $m_{t}$ in the range of eq. (1), the tree-level predictions for $m_{h}$ and $m_{H}$ can be badly violated, and so for the related inequalities. The other free parameter is $m_{\tilde{q}}$, but the dependence on it is much milder. To illustrate the impact of these results, we display in fig. 1 contours of the maximum allowed value of $m_{h}$ (reached for $m_{A} \rightarrow \infty$ ), in the $\left(m_{t}, \tan \beta\right)$ and $\left(m_{t}, m_{\tilde{q}}\right)$ planes, fixing $m_{\tilde{q}}=1 \mathrm{TeV}$ and $\tan \beta=m_{t} / m_{b}$, respectively. In the following, when making numerical examples we shall always choose the representative value $m_{\tilde{q}}=1 \mathrm{TeV}$. To plot different quantities of physical interest in the $\left(m_{A}, \tan \beta\right)$ plane, which is going to be the stage of the following phenomenological discussion, one needs to fix also the value of $m_{t}$. In this paper, whenever an illustration of the $m_{t}$ dependence is needed, we work with the two representative values $m_{t}=120,160 \mathrm{GeV}$, which are significantly different but well within the range of eq. (11). Otherwise, we work with the single representative value $m_{t}=140 \mathrm{GeV}$. As an example, we show in figs. $2-4$ contours of constant $m_{h}, m_{H}$, and $m_{H^{ \pm}}$in the $\left(m_{A}\right.$, $\left.\tan \beta\right)$ plane. Here and in the following we vary $m_{A}$ and $\tan \beta$ in the ranges

$$
0 \leq m_{A} \leq 500 \mathrm{GeV}, \quad 1 \leq \tan \beta \leq 50
$$

The effective-potential method allows us to compute also the leading corrections to the trilinear and quadrilinear Higgs self-couplings. A detailed discussion and the full diagrammatic calculation will be given elsewhere. 
Here we just give the form of the leading radiative corrections to the trilinear $h A A, H A A$, and $H h h$ couplings, which will play an important role in the subsequent discussion of Higgs-boson branching ratios. One finds [23,24]

$\lambda_{h A A}=\lambda_{h A A}^{0}+\Delta \lambda_{h A A}, \quad \lambda_{H A A}=\lambda_{H A A}^{0}+\Delta \lambda_{H A A}, \quad \lambda_{H h h}=\lambda_{H h h}^{0}+\Delta \lambda_{H h h}$,

where

$$
\begin{gathered}
\lambda_{h A A}^{0}=-\frac{i g m_{Z}}{2 \cos \theta_{W}} \cos 2 \beta \sin (\beta+\alpha), \\
\lambda_{H A A}^{0}=\frac{i g m_{Z}}{2 \cos \theta_{W}} \cos 2 \beta \cos (\beta+\alpha), \\
\lambda_{H h h}^{0}=-\frac{i g m_{Z}}{2 \cos \theta_{W}}[2 \sin (\beta+\alpha) \sin 2 \alpha-\cos (\beta+\alpha) \cos 2 \alpha],
\end{gathered}
$$

and, neglecting the bottom Yukawa coupling and the $D$-term contributions to squark masses

$$
\begin{gathered}
\Delta \lambda_{h A A}=-\frac{i g m_{Z}}{2 \cos \theta_{W}} \frac{3 g^{2} \cos ^{2} \theta_{W}}{8 \pi^{2}} \frac{\cos \alpha \cos ^{2} \beta}{\sin ^{3} \beta} \frac{m_{t}^{4}}{m_{W}^{4}} \log \frac{m_{\tilde{q}}^{2}+m_{t}^{2}}{m_{t}^{2}}, \\
\Delta \lambda_{H A A}=-\frac{i g m_{Z}}{2 \cos \theta_{W}} \frac{3 g^{2} \cos ^{2} \theta_{W}}{8 \pi^{2}} \frac{\sin \alpha \cos ^{2} \beta}{\sin ^{3} \beta} \frac{m_{t}^{4}}{m_{W}^{4}} \log \frac{m_{\tilde{q}}^{2}+m_{t}^{2}}{m_{t}^{2}}, \\
\Delta \lambda_{H h h}=-\frac{i g m_{Z}}{2 \cos \theta_{W}} \frac{3 g^{2} \cos ^{2} \theta_{W}}{8 \pi^{2}} \frac{\cos ^{2} \alpha \sin \alpha}{\sin ^{3} \beta} \frac{m_{t}^{4}}{m_{W}^{4}}\left(3 \log \frac{m_{\tilde{q}}^{2}+m_{t}^{2}}{m_{t}^{2}}-2 \frac{m_{\tilde{q}}^{2}}{m_{\tilde{q}}^{2}+m_{t}^{2}}\right) .
\end{gathered}
$$

Notice that, besides the obvious explicit dependence, in eqs. (21)-(27) there is also an important implicit dependence on $m_{t}$ and $m_{\tilde{q}}$, via the angle $\alpha$, which is determined from the mass matrix of eqs. (14)-(16). We also emphasize that neglecting the $D$-terms in the stop and sbottom mass matrices is guaranteed to give accurate results only for $m_{t} \gg m_{Z}$. For $m_{t} \sim m_{Z}$, one should make sure that the inclusion of $D$-terms does not produce significant modifications of our results. In the case of the $h$ and $H$ masses, and of the mixing angle $\alpha$, complete formulae are available, and this check can be easily performed. In the case of the $h A A, H A A$ and $H h h$ couplings, complete formulae are not yet available. For the phenomenologically most important coupling at the LHC and SSC, $\lambda_{H h h}$, we have explicitly checked that the inclusion of $D$-terms does not produce important modifications of our results. 
Finally, one should consider Higgs couplings to vector bosons and fermions. Tree-level couplings to vector bosons are expressed in terms of gauge couplings and of the angles $\beta$ and $\alpha$. The most important part of the radiative corrections is taken into account by using one-loop-corrected formulae to determine $\alpha$ from the input parameters. Other corrections are at most of order $g^{2} m_{t}^{2} / m_{W}^{2}$ and can be safely neglected for our purposes. Tree-level couplings to fermions are expressed in terms of the fermion masses and of the angles $\beta$ and $\alpha$. In this case, the leading radiative corrections can be taken into account by using the one-loop-corrected expression for $\alpha$ and running fermion masses, evaluated at the scale $Q$ which characterizes the process under consideration. This brings us to the discussion of the renormalization group evolution of the top and bottom Yukawa couplings in the MSSM. As boundary conditions, we assume as usual that $m_{t}\left(m_{t}\right)=m_{t}$ and $m_{b}\left(m_{b}\right)=m_{b}$, with $m_{b}=4.8 \mathrm{GeV}$ and $m_{t}$ numerical input parameters. As stated in the Introduction, we assume in this paper that all supersymmetric particles are heavy. Then, since we want to compute Higgs-boson production cross-sections and branching ratios, we are interested in the standard renormalization group evolution of $h_{t}(Q)\left[h_{b}(Q)\right]$ from $Q=m_{t}\left[Q=m_{b}\right]$ to $Q \simeq m_{H^{ \pm}}, m_{H}$, which is dominated by gluon loops.

To illustrate the behaviour of the Higgs couplings to vector bosons and fermions, as functions of the input parameters, we show in figs. 5-7 contours in the $\left(m_{A}, \tan \beta\right)$ plane of some of the correction factors appearing in table 1 .

\section{LEP limits and implications}

In this section, we briefly summarize the implications of the previous results on MSSM Higgs boson searches at LEP I and LEP II. Partial results were already presented in refs. 21,22].

As already clear from tree-level analyses, the relevant processes for MSSM Higgs boson searches at LEP I are $Z \rightarrow h Z^{*}$ and $Z \rightarrow h A$, which play a complementary role, since their rates are proportional to $\sin ^{2}(\beta-\alpha)$ and $\cos ^{2}(\beta-\alpha)$, respectively. An important effect of radiative corrections 23 is to allow, for some values of the parameters, the decay $h \rightarrow A A$, which would be kinematically forbidden according to tree-level formulae. Experimental limits which take radiative corrections into account have by now been obtained by the four LEP collaborations [25], using different methods to present and 
analyse the data, and different ranges of parameters in the evaluation of radiative corrections. A schematic representation of the presently excluded region of the $\left(m_{A}, \tan \beta\right)$ plane, for the standard parameter choices discussed in sect. 2 , is given in fig. 8 , where the solid lines correspond to our naïvef extrapolation of the exclusion contour given in the first of refs. 225]. For a discussion of the precise experimental bounds, we refer the reader to the above-mentioned experimental publications.

The situation in which the impact of radiative corrections is most dramatic is the search for MSSM Higgs bosons at LEP II. At the time when only tree-level formulae were available, there was hope that LEP could completely test the MSSM Higgs sector. According to tree-level formulae, in fact, there should always be a CP-even Higgs boson with mass smaller than $(h)$ or very close to $(H) m_{Z}$, and significantly coupled to the $Z$ boson. However, as should be clear from the previous section, this result can be completely upset by radiative corrections. A detailed evaluation of the LEP II discovery potential can be made only if crucial theoretical parameters, such as the top-quark mass and the various soft supersymmetry-breaking masses, and experimental parameters, such as the centre-of-mass energy, the luminosity and the $b$-tagging efficiency, are specified. Taking for example $\sqrt{s}=190 \mathrm{GeV}$, $m_{t} \gtrsim 110 \mathrm{GeV}$, and our standard values for the soft supersymmetry-breaking parameters, in the region of $\tan \beta$ significantly greater than 1 , the associated production of a $Z$ and a CP-even Higgs can be pushed beyond the kinematical limit. Associated $h A$ production could be a useful complementary signal, but obviously only for $m_{h}+m_{A}<\sqrt{s}$. Associated $H A$ production is typically negligible at these energies. To give a measure of the LEP II sensitivity, we plot in fig. 8 contours associated with two benchmark values of the total cross-section $\sigma\left(e^{+} e^{-} \rightarrow h Z^{*}, H Z^{*}, h A, H A\right)$. The dashed lines correspond to $\sigma=0.2 \mathrm{pb}$ at $\sqrt{s}=175 \mathrm{GeV}$, which could be seen as a rather conservative estimate of the LEP II sensitivity. The dash-dotted lines correspond to $\sigma=0.05 \mathrm{pb}$ at $\sqrt{s}=190 \mathrm{GeV}$, which could be seen as a rather optimistic estimate of the LEP II sensitivity. In computing these cross-sections, we have taken into account the finite $Z$ width, but we have

${ }^{4}$ We fitted the experimental exclusion contours, corresponding to $m_{t}=140 \mathrm{GeV}$ and the other parameters as chosen here, with two numerical values for $\Gamma\left(Z \rightarrow h Z^{*}\right)$ and $\Gamma(Z \rightarrow h A)$. We have then computed radiative corrections for the two values of $m_{t}$ considered here, assuming that the variations in experimental efficiencies are small enough not to affect our results significantly. 
neglected initial state radiation, which leads to suppression near threshold. A more accurate estimate of the LEP II sensitivity can be found in ref. [26]. Of course, one should keep in mind that there is, at least in principle, the possibility of further extending the maximum LEP energy up to values as high as $\sqrt{s} \simeq 230-240 \mathrm{GeV}$, at the price of introducing more (and more performing) superconducting cavities into the LEP tunnel [27].

In summary, a significant region of the parameter space for MSSM Higgses could be beyond the reach of LEP II, at least if one sticks to the reference centre-of-mass energy $\sqrt{s} \lesssim 190 \mathrm{GeV}$. The precise knowledge of this region is certainly important for assessing the combined discovery potential of LEP and $\mathrm{LHC} / \mathrm{SSC}$, but it does not affect the motivations and the techniques of our study, devoted to LHC and SSC searches. Whether or not a Higgs boson will be found at LEP in the future, we want to investigate the possibilities of searching for all the Higgs states of the MSSM at large hadron colliders, in the whole region of parameter space which is not already excluded at present. Even if a neutral Higgs boson is found at LEP, with properties compatible with the SM Higgs boson within the experimental errors, it will be impossible to exclude that it belongs to the MSSM sector. The LHC and SSC could then play a role in investigating its properties and in looking for the remaining states of the MSSM.

Similar considerations can be made for charged-Higgs searches at LEP II with $\sqrt{s} \lesssim 190 \mathrm{GeV}$. In view of the $\beta^{3}$ threshold factor in $\sigma\left(e^{+} e^{-} \rightarrow H^{+} H^{-}\right)$, and of the large background from $e^{+} e^{-} \rightarrow W^{+} W^{-}$, it will be difficult to find the $H^{ \pm}$at LEP II unless $m_{H^{ \pm}} \lesssim m_{W}$, and certainly impossible unless $m_{H^{ \pm}}<\sqrt{s} / 2$. We also know [23,20] that for generic values of the parameters there are no large negative radiative corrections to the charged-Higgs mass formula, eq. (6). A comparison of figs. 4 and 8 indicates that there is very little hope of finding the charged Higgs boson of the MSSM at LEP II (or, stated differently, the discovery of a charged Higgs boson at LEP II would most probably rule out the MSSM).

\section{Branching ratios}




\subsection{Neutral Higgs bosons}

The branching ratios of the neutral Higgs bosons of the MSSM were systematically studied in ref. [12], using the tree-level formulae for masses and couplings available at that time (previous work on the subject is summarized in ref. [3]). Here we present a systematic study which includes the radiative corrections described in sect. 2. As usually done for the SM Higgs boson, we consider the two-body decay channels

$$
h, H, A \longrightarrow c \bar{c}, b \bar{b}, t \bar{t}, \tau^{+} \tau^{-}, g g, \gamma \gamma, W^{*} W^{*}, Z^{*} Z^{*}, Z \gamma
$$

For consistency, we must also consider decays with one or two Higgs bosons in the final state

$$
h \rightarrow A A, \quad H \rightarrow h h, A A, Z A, \quad A \rightarrow Z h .
$$

On the other hand, we neglect here possible decays of MSSM Higgs bosons into supersymmetric particles: as previously stated, we consistently assume a heavy spectrum of $R$-odd particles, so that only $R$-even ones can be kinematically accessible in the decays of $h, H$, and $A$. We perform our study in the framework of MSSM parameter space, with the representative parameter choices illustrated in sect. 2 . The effects of changing the mass of the top quark, and the sensitivity to squark masses in the high-mass region, will also be briefly discussed.

The partial widths for the decays of eq. (28) that correspond to treelevel diagrams can be obtained from the corresponding formulae for the SM Higgs boson (for a summary, see ref. [3]), by simply multiplying the various amplitudes by the supersymmetric correction factors listed in table 1 . For decays that are described by loop diagrams, however, in the MSSM one has to include some contributions that are absent in the SM. Diagrams corresponding to the exchange of $R$-odd supersymmetric particles give negligible contributions to the corresponding partial widths, in the limit of heavy supersymmetric-particle masses that we have chosen for our analysis (in accordance with intuitive ideas about decoupling). One must also include the charged-Higgs loop contributions to the $\gamma \gamma$ and $Z \gamma$ final states. When considering instead the processes of eq. (29), we improve the tree-level formulae

\footnotetext{
${ }^{5}$ Also, the partial widths for the decays $h, H, A \rightarrow \gamma \gamma$ were affected by numerical errors.
} 
of ref. [3] not only with the self-energy corrections to the mixing angle $\alpha$, but also with the vertex corrections of eqs. (21)-(27).

QCD [28] and electroweak [29] radiative corrections to the fermion-antifermion and the $W W, Z Z$ channels have been recently computed for the SM Higgs boson, $\varphi$. They have been found to be small (less than $\sim 20 \%$ ), with the exception of the QCD corrections to the decays into charm and bottom quark pairs, which are large because of running-quark-mass effects. We then included the QCD corrections as described in ref. [14]. One may also wonder whether running-mass effects induced by the large top Yukawa coupling could give further important effects. However, one can easily see that these effects give corrections which are certainly less than $20 \%$.

The QCD correction to $\varphi \rightarrow \gamma \gamma$ is also available, and known to be negligibly small [30]. Sizeable QCD corrections are found, however, for the decay $\varphi \rightarrow g g$ [31]. Although this effect is not important for the branching ratio study, since $\varphi \rightarrow g g$ is neither the dominant decay mode nor a useful channel for detection, it still has to be included in the production cross-section of $h$ via the two-gluon fusion mechanism.

Another general and well-known property of the MSSM is that the selfinteractions of the Higgs bosons are controlled, modulo the logarithmic corrections discussed in sect. 2, by the $S U(2)$ and $U(1)$ gauge couplings. Therefore, the total widths of all MSSM Higgs bosons, displayed in fig. 9, stay below $10 \mathrm{GeV}$ in the whole parameter space we have considered.

The most important branching ratios for the neutral MSSM Higgs bosons are shown, as a function of the mass of the decaying particle, in figs. 10-12. To avoid excessive proliferation of figures, we consider the two representative values

$$
\tan \beta=1.5,30,
$$

and for each of these we vary $m_{A}$ between the experimental lower bound of fig. $8\left(m_{A} \simeq 59 \mathrm{GeV}\right.$ for $\tan \beta=1.5, m_{A} \simeq 44 \mathrm{GeV}$ for $\left.\tan \beta=30\right)$ and the upper bound of eq. (20), assuming $m_{t}=140 \mathrm{GeV}$ and $m_{\tilde{q}}=1 \mathrm{TeV}$.

We consider first the branching ratios of $h$ (fig. 10). We can clearly see the effect of radiative corrections on the allowed range of $m_{h}$ for the given values of $\tan \beta$. For $m_{A} \lesssim 25 \mathrm{GeV}$, the decay $h \rightarrow A A$ can be kinematically allowed and even become the dominant mode. This decay channel was important at LEP I, but since the corresponding region of parameter space is already excluded by experiment, this decay mode does not appear in fig. 10 . 
The dominant decay mode is then $h \rightarrow b \bar{b}$, whereas the $\tau^{+} \tau^{-}$mode has a branching ratio of about $8 \%$ throughout the relevant part of the parameter space. In fig. 10, one immediately notices the rather steep slopes for the $c \bar{c}$ and $\gamma \gamma$ branching ratios plotted versus $m_{h}$, with larger effects for larger values of $\tan \beta$ : their origin can be understood by looking at figs. 2 and $5-7$, which show how $m_{h}$ and the $h$ couplings to heavy fermions and vector bosons vary in the $\left(m_{A}, \tan \beta\right)$ plane.

If the SM Higgs boson is in the intermediate mass region, $m_{\varphi}=70-140$ $\mathrm{GeV}$, at large hadron colliders a measurable signal can be obtained via the $\gamma \gamma$ mode. Since the mass of the light Higgs $h$ is indeed below or inside this region, the $\gamma \gamma$ mode is also crucial for the MSSM Higgs search. Furthermore, the $\gamma \gamma$ branching ratio as a function of the Higgs mass exhibits a rather peculiar behaviour, not only for $h$ but also for $H$ and $A$, so a more detailed discussion is in order. The partial width is given by

$$
\Gamma(\phi \rightarrow \gamma \gamma)=\frac{\alpha^{2} g^{2}}{1024 \pi^{3}} \frac{m_{\phi}^{3}}{m_{W}^{2}}\left|\sum_{i} I_{i}^{\phi}\left(\tau_{i}^{\phi}\right)\right|^{2}, \quad \tau_{i}^{\phi}=\frac{4 m_{i}^{2}}{m_{\phi}^{2}},
$$

where $\phi=h, H, A$ and $i=f, W, H, \tilde{f}, \tilde{\chi}$ indicates the contributions from ordinary fermions, charged gauge bosons, charged Higgs bosons, sfermions and charginos, respectively. The functions $I_{\phi}^{i}\left(\tau_{\phi}^{i}\right)$ are given by

$$
\begin{aligned}
I_{f}^{\phi} & =F_{1 / 2}^{\phi}\left(\tau_{f}^{\phi}\right) N_{c f} e_{f}^{2} R_{f}^{\phi}, \\
I_{W}^{\phi} & =F_{1}\left(\tau_{W}^{\phi}\right) R_{W}^{\phi}, \\
I_{H}^{\phi} & =F_{0}\left(\tau_{H}^{\phi}\right) R_{H}^{\phi} \frac{m_{W}^{2}}{m_{\phi}^{2}}, \\
I_{\tilde{f}}^{\phi} & =F_{0}\left(\tau_{\tilde{f}}^{\phi}\right) N_{c f} e_{f}^{2} R_{\tilde{f}}^{\phi} \frac{m_{Z}^{2}}{m_{\tilde{f}}^{2}}, \\
I_{\tilde{\chi}}^{\phi} & =F_{1 / 2}^{\phi}\left(\tau_{\chi}^{\phi}\right) R_{\tilde{\chi}}^{\phi} \frac{m_{W}}{m_{\tilde{\chi}}},
\end{aligned}
$$

where $N_{c f}$ is 1 for (s)leptons and 3 for (s)quarks, and the subscripts of the complex functions $F_{1 / 2}^{S}(\tau), F_{1 / 2}^{P}(\tau), F_{0}(\tau)$, and $F_{1}(\tau)$, which were calculated in ref. [32], indicate the spin of the particles running in the loop. In the case of spin-1/2 particles, the contribution is different for CP-even and CP-odd neutral Higgses. The symbols $R_{i}^{\phi}$ denote the appropriate correction factors 
for the MSSM Higgs couplings: for $i=f, W$ they are given in table 1 , for $i=H, \tilde{f}, \tilde{\chi}$ they can be found, for example, in Appendix $\mathrm{C}$ of ref. [3]. The $W$ contribution dominates the $h \rightarrow \gamma \gamma$ decay rate. The function $F_{1}$ is large at and above $\tau=1$. For the $W$ contribution $\tau=4 m_{W}^{2} / m_{h}^{2}>1$, and increasing $m_{h}$ gives increasing values of $F_{1}$. The steep dependence of the branching ratio on $m_{h}$ is a consequence of the fast change of $\sin ^{2}(\beta-\alpha)$ as $m_{A}$ is increased for fixed $\tan \beta$. This is further enhanced by the fact that the large interval $100 \mathrm{GeV} \lesssim m_{A} \leq 500 \mathrm{GeV}$ is mapped into a very small interval (a few GeV) in $m_{h}$. We elucidate this effect by plotting in fig. 13 the branching ratios of $h$ as a function of $m_{A}$, for the same values of the parameters as in fig. 10 . We can see that the tip of the $g g, c \bar{c}$ and $\gamma \gamma$ curves in fig. 10 is mapped into a long plateau in fig. 13. We can also observe that in a large region of the parameter space the $h \rightarrow \gamma \gamma$ branching ratio has a value somewhat smaller than (but comparable to) the corresponding branching ratio for a SM Higgs of mass $m_{h}$. This is due to the fact that all the $h$ couplings tend to the SM Higgs couplings for $m_{A} \gg m_{Z}$; however, for the $h$ couplings to fermions the approach to the asymptotic value is much slower than for the $h$ couplings to vector bosons, as can be seen from figs. 5 to 7 . In fig. 13, the branching ratios for the $W^{*} W^{*}$ and $Z^{*} Z^{*}$ decays are also plotted, whereas they were omitted in fig. 10 in order to avoid excessive crowding of curves. However, for our parameter choice they have little interest at large hadron colliders, because of the small production rates and the large backgrounds.

The branching ratios of the heavy Higgs boson $H$, depicted in fig. 11, have a rather complicated structure. We make here four remarks.

i) The $\gamma \gamma$ mode has a steeply decreasing branching ratio with increasing $m_{H}$, except at small values of $\tan \beta$ and at the lower kinematical limit of $m_{H}$, where one or more of the $A A, Z A$ and $h h$ decay channels are open. The steep fall of the $\gamma \gamma$ branching ratio at large values of $\tan \beta$ can be easily understood. The partial width $\Gamma(H \rightarrow \gamma \gamma)$ is dominated by the $W$ contribution, proportional to $\cos ^{2}(\beta-\alpha)$. As we can see in fig. $7, \cos ^{2}(\beta-$ $\alpha$ ) decreases very fast, for increasing $m_{H}$, at fixed values of $\tan \beta$. This steep decrease is slightly compensated by the increase of $F_{1}\left(\tau_{W}\right)$ at $\tau_{W} \leq 1$, which has a peak at the $W$ threshold $m_{H}=2 m_{W}$. Another peak in the $\gamma \gamma$ branching ratio is obtained, for small values of $\tan \beta$, at $m_{H}=2 m_{t}$, where the top-quark loop gives the dominant contribution.

ii) The complicated structure in the $H$ branching ratio curves is mainly due to the $H \rightarrow h h$ channel. For $m_{H}<2 m_{t}$, and not too high values 
of $\tan \beta$, this decay mode is dominant whenever kinematics allows. This channel is always open at the lower kinematical limit of $M_{H}$. Increasing $M_{H}$ a little bit, however, it may become strongly suppressed, because for small increasing values of $m_{A}$ the value of $m_{h}$ rises faster than that of $m_{H}$, so that the channel can become kinematically closed. Obviously, for sufficiently high values of $m_{H}$ the channel is always open. At high values of $\tan \beta$, the mass region at the lower kinematical limit where $H \rightarrow h h$ is open becomes smaller and smaller, explaining the presence of the almost vertical line in fig. 11. A further structure is present in this decay channel due to the coupling factor $\lambda_{H h h}$ [see eqs. (24) and (27)]. There are relatively small values of $m_{H}$ at which $\lambda_{H h h}$ accidentally vanishes. Furthermore, for very large values of $m_{H}$ and $\tan \beta$ one has $\alpha \simeq 0, \beta \simeq \pi / 2$, and therefore $\lambda_{H h h} \simeq 0$. Unfortunately, even when it is dominant, this mode has very large backgrounds, so it seems unlikely to give a measurable signal at large hadron colliders. The $H \rightarrow A A$ mode is kinematically allowed only for values of $m_{A}$ below $50-60 \mathrm{GeV}$, in which case it can have a large branching ratio, competing with the one for $H \rightarrow h h$. The $H \rightarrow Z A$ mode is kinematically allowed only in the region of parameter space which is already excluded by the LEP I data.

iii) $H$ can decay at tree level into $Z Z \rightarrow l^{+} l^{-} l^{+} l^{-}$, which is the 'goldplated' signature for the SM Higgs boson. Unfortunately, in the case of $H$ the branching ratio is smaller, and it decreases fast with increasing $\tan \beta$ and/or $m_{A}$. For small $\tan \beta$ and $2 m_{h}<m_{H}<2 m_{t}$, this mode is suppressed by the competition with $H \rightarrow h h$, and this effect is further enhanced by the inclusion of the radiative correction of eq. (27), which typically gives an additional $50 \%$ suppression. Nevertheless, as we shall see in the next section, the four-lepton channel can give a measurable signal in some small region of the parameter space.

iv) The decay into $t \bar{t}$ is dominant above threshold at moderate values of $\tan \beta$. But above $\tan \beta \sim 8$ or so the $b \bar{b}$ mode remains dominant and $\tau^{+} \tau^{-}$ has the typical $\sim 10 \%$ branching ratio.

Finally, we discuss the branching ratios of $A$, shown in fig. 12. The $\gamma \gamma$ one is always small, although at small $\tan \beta$ and slightly below the top threshold, $m_{A} \sim 2 m_{t}$, it reaches a value $\sim 8 \times 10^{-4}$, which may give a measurable signal in a small island of the parameter space. The behaviour of the $A \rightarrow \gamma \gamma$ branching ratio can be easily understood by taking into account that the partial width is dominated by the top loop contribution. Two features are important here. First, the function $F_{1 / 2}(\tau)$ appearing in eq. (32) has a strong 
enhancement at $\tau \sim 1$. Furthermore, the $t \bar{t} A$ coupling gives a suppression factor $1 /(\tan \beta)^{2}$ for increasing values of $\tan \beta$.

At smaller values of $m_{A}$ and $\tan \beta$, there is a substantial branching ratio to $Z h$, which however does not look particularly promising for detection at large hadron colliders, because of the very large $Z b \bar{b}$ background. We can see that all the dominant decay modes of the $A$ boson correspond to channels which are overwhelmed by very large background, except perhaps the $\tau^{+} \tau^{-}$ mode, which, as we shall see in the next section, may give a detectable signal for very high values of $\tan \beta$.

We have studied the neutral Higgs branching ratios also at $m_{t}=120,160,180 \mathrm{GeV}$. Increasing the top mass has two major effects. First, the maximum value of $m_{h}$ increases (see fig. 1). Next, owing to the increased value of the top threshold, the structure generated by the opening of the top decay channel is shifted to higher mass values. We also note that varying $m_{\tilde{q}}$ in the range 0.5-2 TeV has negligible effects on the branching ratio curves of figs. 10-12. Finally, if one chooses $m_{t}$ and $m_{\tilde{q}}$ so large that $m_{h}>130 \mathrm{GeV}$, the $W^{*} W^{*}$ and $Z^{*} Z^{*}$ branching ratios can become relevant also for $h$.

\subsection{Charged Higgs boson}

In the case of the charged Higgs boson, we considered only the two-body decay channels

$$
H^{+} \rightarrow c \bar{s}, \tau^{+} \nu_{\tau}, t \bar{b}, W^{+} h
$$

Tree-level formulae for the corresponding decay rates can be found, for instance, in ref. [3]. Loop-induced decays such as $H^{+} \rightarrow W^{+} \gamma, W^{+} Z$ have very small branching ratios [33] and are not relevant for experimental searches at the LHC and SSC. Radiative corrections to the charged-Higgs-boson mass formula were included according to eqs. (17) and (18). The $H^{+} W^{-} h$ coupling, proportional to $\cos (\beta-\alpha)$, was evaluated with the one-loop corrected value of $\alpha$. The leading QCD corrections to the $H^{+} b \bar{t}$ and $H^{+} s \bar{c}$ vertices were parametrized, following refs. [34], by running quark masses evaluated at a scale $Q \sim m_{H^{ \pm}}$. The resulting branching ratios for the charged Higgs boson are displayed in fig. 14, for $\tan \beta=1.5,30$ and the standard parameter choice $m_{t}=140 \mathrm{GeV}, m_{\tilde{q}}=1 \mathrm{TeV}$. One can see that the dominant factor affecting the branching ratios is the $m_{H^{ \pm}}=m_{t}+m_{b}$ threshold. Above threshold, the $t \bar{b}$ mode is dominant for any value of $\tan \beta$ within the bounds of eq. (20). 
Below threshold, the dominant mode is $\tau^{+} \nu_{\tau}$, with the competing mode $c \bar{s}$ becoming more suppressed for higher values of $\tan \beta$. For small values of $\tan \beta$, the $W^{+} h$ decay mode can also be important, and even dominate, in a limited $m_{H^{ \pm}}$interval, if the $W^{+} h$ threshold opens up before the $t \bar{b}$ one. The exact position of the two thresholds on the $m_{H^{ \pm}}$axis depends of course on $\tan \beta, m_{t}$, and $m_{\tilde{q}}$. It is just a numerical coincidence that in fig. 14a the two thresholds correspond almost exactly. For increasing values of $m_{H^{ \pm}}$ and $\tan \beta$, the numerical relevance of the $W^{+} h$ branching ratio rapidly dis-

appears, because of the $\cos ^{2}(\beta-\alpha)$ suppression factor in the corresponding partial width.

The total charged Higgs boson width is shown, as a function of $m_{H^{ \pm}}$and for $\tan \beta=1.5,3,10,30$, in fig. 9d. Again one can see the effects of the $t \bar{b}$ threshold, and also the $\tan \beta$-dependence of the couplings to fermions. In any case, the charged Higgs width remains smaller than $1 \mathrm{GeV}$ for $m_{H^{ \pm}}<$ $m_{t}+m_{b}$, and smaller than $10 \mathrm{GeV}$ for $m_{H^{ \pm}}<500 \mathrm{GeV}$.

\section{$5 \quad$ Neutral-Higgs production cross-sections}

There is only a limited number of parton-level processes which can give interesting rates for the production of the MSSM neutral Higgs bosons $(\phi=$ $h, H, A)$ at proton-proton supercolliders:

$$
\begin{array}{r}
g+g \rightarrow \phi, \\
q+q \rightarrow q+q+W^{*}+W^{*} \rightarrow q+q+\phi, \\
g+g \text { or } q+\bar{q} \rightarrow b+\bar{b}+\phi, \\
g+g \text { or } q+\bar{q} \rightarrow t+\bar{t}+\phi, \\
q+\bar{q} \rightarrow W(Z)+\phi,
\end{array}
$$

where $q$ denotes any quark flavour. These processes are controlled by the Higgs couplings to heavy quarks and gauge bosons, whose essential features were summarized in table 1 . We briefly discuss here the corresponding cross-sections and the status of their theoretical description, emphasizing the features which are different from the SM case. We shall always adopt the HMRSB structure functions [35] with $\Lambda^{(4)}=190 \mathrm{MeV}$. 
Gluon fusion. In the SM, $g g \rightarrow \phi$ [36] is the dominant production mechanism, the most important diagram being the one associated with the top-quark loop. In the MSSM, this is not always the case, since the correction factors of table 1 give in general suppression for the top contribution and enhancement for the bottom one, and stop and sbottom loops could also play a role.

The leading-order amplitudes for the gluon-fusion processes are determined by the functions of eqs. (32), with top, bottom, stop and sbottom intermediate states. For $m_{\tilde{q}}=1 \mathrm{TeV}$, the squark contributions are very small, owing to the suppression factor $m_{Z}^{2} / m_{\tilde{q}}^{2}$ in the corresponding $I_{\tilde{q}}$ functions. For large values of $\tan \beta$, the bottom contribution can compete with the top one and even become dominant.

QCD corrections to the gluon-fusion cross-section were recently evaluated in ref. [31], for a SM Higgs in the mass region below the heavy-quark threshold. In this region, QCD corrections increase the top contribution by about $50 \%$. To a good approximation, the bulk of QCD corrections can be taken into account by performing the replacement

$$
\sigma_{0}(g g \rightarrow \phi) \longrightarrow \sigma_{0}(g g \rightarrow \phi)\left[1+\left(\frac{11}{2}+\pi^{2}\right) \frac{\alpha_{S}}{\pi}\right]
$$

at the renormalization scale $Q=m_{\phi}$. This calculation, unfortunately, is not valid above the heavy-quark threshold, a region which is relevant for the bottom contribution and for the top contribution to $H, A$ production, when $m_{H}, m_{A}>2 m_{t}$. Even below the heavy-quark threshold, the SM QCD corrections are applicable to $h$ and $H$ production, but not to $A$ production, because of the additional $\gamma_{5}$ factor appearing at the $A q \bar{q}$ vertex. In view of this not completely satisfactory status of QCD corrections, we calculate, conservatively, the top contribution without QCD corrections. However, when discussing the detectability of the different physics signals, we shall take into account the results of ref. 31], when applicable. In the case of the bottom contribution, we use the running $m_{b}$, which leads to suppression.

In fig. 15 we display cross-sections for $g g \rightarrow \phi(\phi=h, H, A)$, as functions of $m_{\phi}$, for $\tan \beta=1.5,3,10,30$ and for LHC and SSC energies. The SM Higgs cross-section is also shown for comparison. For large values of $\tan \beta$ and not too high values of $m_{\phi}$, the cross-sections can be enhanced with respect to the SM value. This effect is due to the enhanced bottom-quark contribution, as apparent from table 1 and fig. 5. The fast disappearance of this effect for 
increasing Higgs masses is due to the fast decrease of the function $F_{1 / 2}^{\phi}\left(\tau_{b}^{\phi}\right)$ as $\tau_{b}^{\phi} \rightarrow 0$. When the neutral Higgs couplings to fermions are SM-like, the gluon-fusion cross-sections approach the SM value, and are always dominated by the top contribution. The changes in the slopes of the curves in fig. 15 are due to the competing top and bottom contributions. In particular, one can notice an important threshold effect, for $m_{A} \sim 2 m_{t}$, in the process $g g \rightarrow A$, which can bring the corresponding cross-section above the SM value for low $\tan \beta$.

As a final remark, we notice that the LHC and SSC curves in fig. 15 have very similar shapes, with a scaling factor which is determined by the gluon luminosity and uniformly increases from $\sim 2.5$ at $m_{\phi} \sim 100 \mathrm{GeV}$ to $\sim 5$ at $m_{\phi} \sim 500 \mathrm{GeV}$.

$W$ fusion. In the SM case, the $W$-fusion mechanism 37] can compete with the gluon-fusion mechanism only for a very heavy $\left(m_{\varphi} \gtrsim 500 \mathrm{GeV}\right)$ Higgs boson, owing to the enhanced $W_{L} W_{L} \varphi$ coupling and to the relative increase of the quark number-densities. In the MSSM, the correction factors for the couplings to vector boson pairs (see table 1 and fig. 7) are always smaller than 1, so that the MSSM $W$-fusion cross-sections are always smaller than the SM one.

We illustrate this in fig. 16, where $W$-fusion cross-sections for $h$ and $H$ are displayed, for the same $\tan \beta$ and $\sqrt{s}$ values as in fig. 15. For both $h$ and $H$, the SM cross-section is approached from below in the regions of parameter space where $\sin ^{2}(\beta-\alpha) \rightarrow 1$ and $\sin ^{2}(\beta-\alpha) \rightarrow 0$, respectively. In figs. $16 \mathrm{~b}$ and $16 \mathrm{~d}$, for $m_{A} \rightarrow 0$ there is a positive lower bound on $\sin ^{2}(\beta-\alpha)$ (see fig. 7), reflecting the fact that at the tree level $\alpha \rightarrow-\beta$ in this limit, so the SM value is actually never reached. For increasing $m_{H}$, one can notice the fast decoupling of $H$ from $W$-pairs, as already observed when discussing the total width. In leading order, $A$ does not couple to $W$-pairs. A non-vanishing cross-section could be generated at one loop, but such a contribution is completely negligible, since even for $h$ and $H$ the $W$-fusion cross-section is small ( $\lesssim 20 \%$ ) compared with the gluon-fusion cross-section. Finally, we observe that the LHC and SSC cross-sections of fig. 16 differ by an overall factor $\sim 3$ in the phenomenologically relevant region, $m_{\phi}=70-140$ $\mathrm{GeV}$. 
Associated $b \bar{b} \phi$ production. This mechanism is unimportant in the $\mathrm{SM}$, since its cross-section is too small to give detectable signals [38]. In the MSSM model, however, for large values of $\tan \beta$ the $b \bar{b} \phi$ couplings can be strongly enhanced. Then for not too high values of the Higgs masses, a significant fraction of the total cross-section for neutral Higgs bosons can be due to this mechanism.

The associated $b \bar{b} \phi$ production involves two rather different mass scales, $m_{\phi}>>m_{b}$, therefore at higher orders large logarithmic corrections of order

$$
\alpha_{s}^{n} \ln ^{n}\left(\frac{m_{\phi}}{m_{b}}\right)
$$

may destroy the validity of the Born approximation, depending on the value of $m_{\phi}$. One needs an improved treatment where these logarithms are resummed to all orders. The origin of these logarithms is well understood. Part of them come from configurations where the gluons are radiated collinearly by nearly on-shell bottom quarks, which are obtained by splitting the initial gluons into a $b \bar{b}$ pair. This type of logarithms are responsible for the QCD evolution of the effective $b$-quark density within the proton: they were carefully analysed and resummed to all orders, and it was found [39] that the corrections are positive and increase with the Higgs-boson mass. A second subset of logarithms lead to running quark mass effects. An analysis where both effects are treated simultaneously is still missing. In view of this ambiguity, we interpolated the existing results by using the Born approximation with the bottom quark mass adjusted to the fixed value $m_{b}=4 \mathrm{GeV}$. However, one should keep in mind that the theoretical estimate in this case has a large (factor of 2) uncertainty.

In fig. 17 we display cross-sections for associated $b \bar{b} \phi$ production, for the same $\tan \beta$ and $\sqrt{s}$ values as in fig. 15. Comparing the cross-sections of figs. 15 and 17, we can see that the $h b \bar{b}$ cross-section can give at most a $20 \%$ correction to the total $h$ cross-section. The $H b \bar{b}$ and $A b \bar{b}$ cross-sections, however, can be even larger than the corresponding gluon-fusion cross-sections for $\tan \beta \gtrsim 10$. Comparing the LHC and SSC curves of fig. 17, one can notice a rescaling factor varying from $\sim 3$ to $\sim 8$ in the $m_{\phi}$ region from 60 to 500 $\mathrm{GeV}$.

Associated $W \phi(Z \phi)$ production. This mechanism [40] is the hadron collider analogue of the SM Higgs production mechanism at LEP, with the 
difference that at hadron colliders $W \phi$ production is more important than $Z \phi$ production. In the $Z \phi$ case, the event rate at the $\mathrm{LHC}$ and $\mathrm{SSC}$ is too low to give a detectable signal, both in the SM and (consequently) in the MSSM. The $W \phi$ mechanism has considerable importance at the LHC for $\phi=h, H$ and in the Higgs mass range $m_{\phi}=70-140 \mathrm{GeV}$, where a measurable signal may be obtained from final states consisting of two isolated photons and one isolated lepton. The calculation of the cross-section is well understood, including the QCD corrections, since it has a structure similar to the Drell-Yan process, with the same next-to-leading-order corrections (for a recent study concerning the numerical importance of the QCD corrections see ref. 411). The QCD corrections are positive, and amount to about $12 \%$ if one chooses $Q^{2}=\hat{s}$ as the scale of $Q^{2}$ evolution. The production crosssections of $h$ and $H$ are obtained by rescaling the SM model cross-section by the appropriate correction factors given in table 1 .

In fig. 18, cross-sections for $W h$ and $W H$ are displayed, as functions of corresponding Higgs masses, for the same $\tan \beta$ and $\sqrt{s}$ values as in fig. 15 . Since the SUSY correction factors are the same, the approach to the SM case and the irrelevance of $W A$ production can be described in the same way as for the $W$-fusion mechanism.

In the phenomenologically relevant region, $m_{\phi}=70-140 \mathrm{GeV}$, the scaling factor between the LHC and SSC curves is $\sim 2.5$.

Associated $t \bar{t} \phi$ production. In the SM, the Born cross-section formula for this process is the same as for the $b \bar{b} \varphi$ case [38]. In the MSSM case, one just needs to insert the appropriate SUSY correction factors, as from table 1. Note, however, that the leading-order QCD calculation is more reliable in this case, since in the $t \bar{t} \phi$ case one does not have two very different physical scales when $m_{\phi}$ is in the intermediate mass region. The next-toleading QCD corrections are not known, therefore the Born cross-section still suffers from a relatively large $(\sim 50 \%)$ scale ambiguity.

In fig. 19, the production cross-sections for $t \bar{t} \phi(\phi=h, H, A)$ are plotted, as functions of the corresponding Higgs mass, for the same $\tan \beta$ and $\sqrt{s}$ values as in fig. 15. In general, the MSSM cross-sections are smaller than the SM one, which is approached in the limit in which the $t \bar{t} \phi$ coupling becomes SM-like. A possible exception is the $t \bar{t} H$ cross-section for small values of $m_{A}$ and $\tan \beta$, since in this case the corresponding coupling can be slightly enhanced with respect to the SM one. 
In the phenomenologically relevant range, $m_{\phi}=70-140 \mathrm{GeV}$, the rescaling factor between the LHC and SSC curves in fig. 19 varies from $\sim 6$ to $\sim 7$.

In the phenomenologically allowed range of eq. (1), the top-mass dependence of the cross-sections of figs. 15-19 is not negligible, but it does not change qualitatively the previous considerations. The largest effect comes from the increase of the upper limit on $m_{h}$ for increasing top mass (see fig. 1). This induces a shift in the limiting values for the $h$ and $H$ production cross-sections. There are also obvious kinematical top-mass effects in the gluon-fusion mechanism and in the $t \bar{t} \phi$ mechanism, which are well understood from SM studies [14]. In the MSSM, additional effects are given by the radiative corrections to the relevant Higgs couplings, which were discussed in sect. 2.

\section{Physics signals}

\subsection{Neutral Higgs bosons}

We now calculate the rates for a number of processes that could provide evidence for one or more of the neutral MSSM Higgs bosons at the LHC and SSC, and we summarize our results with the help of contour plots in the $\left(m_{A}, \tan \beta\right)$ plane. We consider production cross-sections, folded with branching ratios, for the following signals:

- two isolated photons;

- one isolated lepton and two isolated photons;

- four isolated charged leptons;

- a pair of tau leptons.

In the SM case, the first two signals are relevant for the region of intermediate Higgs mass, $70 \mathrm{GeV}<m_{\phi}<140 \mathrm{GeV}$, the third one is the so-called 'goldplated' signal in the high-mass region $130 \mathrm{GeV}<m_{\phi}<800 \mathrm{GeV}$, and the $\tau^{+} \tau^{-}$signal appears to be hopelessly difficult. 
In a complete phenomenological study, one would like to determine precisely the statistical significance of the different physics signals. This would require, besides the computation of total signal rates, the calculation of the backgrounds, the determination of the efficiencies (for both signals and backgrounds) due to kinematical cuts and detector effects, the optimization of the kinematical cuts to achieve the best signal/background ratio, etc. Such a complete analysis would require the specification of several detector and machine parameters, and goes beyond the aim of the present paper. Instead, we try here to present total rates for well-defined physics signals, in a form which should be useful as a starting point for dedicated experimental studies.

As the only exception, to illustrate with an example how our results can be used to establish the statistical significance of a given physics signal in a given detector, we shall describe the case of the 'two-isolated-photons' signal, using the results of recent simulation works. A similar procedure should be adopted for any other physics signal, detector, and collider, once complete results of simulation works are available. In many cases, the existing results from previous background and simulation studies, carried out for the SM, can also be used to draw conclusions concerning the MSSM case. We mention, however, two important differences: 1$)$ the total widths of $H$ and $A$ remain small even in the high-mass region; 2) for large $\tan \beta$, the number of signal events in the $\tau^{+} \tau^{-}$final state is significantly higher than in the SM case.

Inclusive two-photon channel. In fig. 20 we display cross-sections times branching ratios for the inclusive production of $\phi=h, H, A$, followed by the decay $\phi \rightarrow \gamma \gamma$, as functions of $m_{\phi}$, and for the same parameter choices and energies as in fig. 15. We sum the contributions of the gluon-fusion, $W$ fusion, and $b \bar{b} \phi$ mechanisms. For comparison, the SM value is also indicated. The QCD corrections of ref. 31] are not included, for the reasons explained in the previous section. In the case of $h$ and $H$, the signal rates are always smaller than in the SM, and approach the SM values at the upper and lower edge of the allowed $m_{h}$ amd $m_{H}$ ranges, respectively. The rather steep slope characterizing the approach to the SM limit, for varying Higgs mass and fixed $\tan \beta$, is a reflection of the property of the branching ratios discussed in sect. 4. Also the structure in figs. $20 \mathrm{~b}$ and $20 \mathrm{e}$ can be attributed to the threshold behaviour of the $H \rightarrow h h$ channel. The signal rate for the CP-odd $A$ boson is extremely small for $\tan \beta \gtrsim 3$. However, we observe that in a small region of the parameter space, for $m_{A}$ just below $2 m_{t}$ and $\tan \beta \lesssim 3$, 
the rate can become larger than the SM one: nevertheless, in general it is still too low to produce a detectable signal, unless one chooses $\tan \beta \sim 1$ and $m_{A} \sim 2 m_{t}$.

For the inclusive two-photon channel, the results of detailed simulations of signal and SM background are now available, for some of the LHC detector concepts [42,16, 43, 44]. For illustrative purposes, in the following example we shall follow the treatment of ref. 43. In the mass range $m_{\phi}=80-150 \mathrm{GeV}$, and assuming $10^{5} \mathrm{pb}^{-1}$ integrated luminosity, this LHC simulation considers a fairly wide range of detector performances, which affect the significance of the signal. For an energy resolution $\Delta E / E=[2 \% / \sqrt{E(\mathrm{GeV})}]+0.5 \%$, ref. [43] obtains a $10^{4}$ efficiency for rejecting jets faking an isolated photon in the relevant $p_{T}$ region. Applying standard kinematical cuts, this simulation finds $\sim 40-50 \%$ kinematical acceptance, with an additional $\sim 30-40 \%$ loss due to isolation cuts and reconstruction efficiency for the isolated photons. Typically, for a SM Higgs with $m_{\varphi} \sim 100 \mathrm{GeV}$, one obtains $\sim 10^{3}$ signal events over $\sim 10^{4}$ background events, corresponding to a statistical significance $S / \sqrt{B} \sim 10$. More generally, ref. 43. determined the statistical significance of the signal for given values of the generic Higgs mass $m_{\phi}$ and of the signal rate $\sigma \cdot B R(\phi \rightarrow \gamma \gamma)$ (see fig. 21). In our opinion, this is an excellent way of summarizing the simulation work, since it gives the possibility of studying alternatives to the SM case, and in particular the MSSM. The dashed line in fig. 21 corresponds to the signal for the SM Higgs, which includes both the gluon-fusion and the $W$-fusion production mechanisms, and also the QCD corrections of ref. 31. One can see from fig. 21 that for such optimistic detector parameters there is some margin for detecting smaller rates than in the SM. Clearly the SUSY Higgs search further enhances the need for the best possible $m_{\gamma \gamma}$ resolution and $\gamma$-jet rejection.

In extending the SM analysis to the MSSM, one should pay attention to the applicability of the QCD corrections of ref. 31 to the gluon-fusion cross-section. We have checked that in the phenomenologically relevant region, which corresponds to $h$ or $H$ in the intermediate mass range, and to signal rates within an order of magnitude from the SM one, the gluon-fusion mechanism is dominated by the top-quark loop. Since in this region the gluon-fusion mechanism accounts for $\sim 80 \%$ of the total cross-section, and the correction is roughly a multiplicative factor 1.5 , as a rule of thumb we can take it into account by multiplying the total cross-section by a factor 
$\sim 1.4$.

In fig. 22 we show contour plots in the $\left(m_{A}, \tan \beta\right)$ plane, corresponding to fixed values of $\sigma \cdot B R(\phi \rightarrow \gamma \gamma)(\phi=h, H)$. QCD corrections have been included according to ref. [31]. The region where the rate is large enough to promise a measurable signal is rather large for $h$, is concentrated in a small strip for $H$, and is possibly a very small area, just below $m_{A}=2 m_{t}$ and just above $\tan \beta=1$, for $A$. In our representative example 433, we can now evaluate the statistical significance of the 'two-isolated-photons' signal at any point of the $\left(m_{A}, \tan \beta\right)$ plane, by just combining the information contained in figs. $22,2,3$, and 21. In the case of $h$ searches, and for $m_{h} \gtrsim 90 \mathrm{GeV}$, a signal rate beyond $40 \mathrm{fb}$ should give detectable signals. A signal rate of 30 $\mathrm{fb}$ is the borderline of detectability for one year of running, and signal rates below $20 \mathrm{fb}$ appear extremely difficult to detect. In the case of $H$, which has higher mass, a signal rate of $20 \mathrm{fb}$ appears to be the borderline of what can be achieved in one year of running. In the case of $A$, the interesting mass region is $m_{A} \sim 2 m_{t}$ : for $m_{A}=250 \mathrm{GeV}$, and taking $\sigma \cdot B R(A \rightarrow \gamma \gamma)=3 \mathrm{fb}$ as a plausible discovery limit at the LHC [43, a signal for $A \rightarrow \gamma \gamma$ will be found only if $\tan \beta \lesssim 1.5$.

One isolated lepton and two isolated photons. This signal can come from either $W \phi$ or $t \bar{t} \phi$ production. In the latter case, two or more isolated jets are also produced. The physics signals from $W \phi$ production are particularly important at the LHC, and were studied in ref. 45. The importance of the physics signals from $t \bar{t} \phi$ production was recently emphasized in ref. 46. The production rates, multiplied by the $\phi \rightarrow \gamma \gamma$ branching ratio, are shown in fig. 23. We can see that, similarly to the inclusive $\gamma \gamma$ channel, the rates for $W h, W H, t \bar{t} h, t \bar{t} H$ are always smaller than the SM value, which represents the boundary curve in the limit of large $\tan \beta$ and large (small) $m_{A}$ for $h(H)$. From figs. $23 \mathrm{~g}$ and $23 \mathrm{j}$ one can see that $t \bar{t} A$ production can give a $l l \gamma$ signal larger than in the SM for small $\tan \beta$ and near the $m_{A}=2 m_{t}$ threshold, but even in this case the rate appears to be too small for detection. We emphasize that the production rates shown in fig. 23 do not include the branching ratios of leptonic $W$ and semileptonic $t$ decays. If top decays are as in the SM, one should still include a combinatorial factor of 2 , coming from the fact that both top and antitop can decay semileptonically. On the other hand, in the MSSM there is the possibility of $t \rightarrow b H^{+}$decays, where the subsequent $H^{+}$decay cannot produce a direct lepton $l=e, \mu$. We shall 
take this possibility into account in the following, but its impact on the detectability of the $l \gamma \gamma$ signal is rather small. The only case in which this effect is not completely negligible is for $H$, when $m_{A} \lesssim 100 \mathrm{GeV}$ and $\tan \beta \lesssim 4$ or $\tan \beta \gtrsim 10$, in which case the $t \rightarrow b H^{+}$branching ratio can play a role.

From parton-level simulations [42,47,48], for a SM Higgs of about 100 $\mathrm{GeV}$, one typically obtains $\sim(12+15)$ and $\sim(3+11) l \gamma \gamma$ signal events at the LHC and SSC, respectively. Here we assumed $10^{5} \mathrm{pb}^{-1}$ of integrated luminosity for the LHC and $10^{4} \mathrm{pb}^{-1}$ for the SSC. The quoted numbers separately show the contributions from $W \varphi$ and $t \bar{t} \varphi$ production. Furthermore, they include losses due to acceptances $(\sim 30 \%)$, and lepton and photon detection efficiencies $\left[\epsilon \sim(0.9)^{3}\right]$. The total background is roughly $20-30 \%$ of the signal and is dominated by the irreducible $W \gamma \gamma$ and $t \bar{t} \gamma \gamma$ contributions. There are many different contributions to the reducible background $(b \bar{b} g, b \bar{b} \gamma, b \bar{b} \gamma \gamma, W j \gamma, \ldots)$. Parton-level simulations indicate that they can be suppressed well below the irreducible background, provided one assumes, as for the inclusive $\gamma \gamma$ case, excellent detector performances: a $\gamma$-jet rejection factor $\gtrsim 3 \times 10^{3}$ and a suppression factor $\gtrsim 7$ for the leptons from $b$-decays after isolation cuts.

Clearly, there is very little margin (a factor of 2?) to be sensitive to signal rates smaller than in the SM. In fig. 24, we show contour plots corresponding to fixed values for the quantity

$$
\begin{aligned}
L_{\phi} & \equiv[\sigma \cdot B R(l \gamma \gamma)]_{\phi} \\
& =[2 \sigma(t \bar{t} \phi) \cdot B R(t \rightarrow W b)+\sigma(W \phi)] \cdot B R(\phi \rightarrow \gamma \gamma) \cdot B R(W \rightarrow l \nu),
\end{aligned}
$$

for the same choice of $m_{t}$ and $m_{\tilde{q}}$ as in fig. 15, and for LHC and SSC energies. In eq. (40), $l=e, \mu$ and we have not considered the strongly suppressed possibility of getting a light charged lepton from both top and antitop.

The four-lepton channel. The channel $\varphi \rightarrow Z^{*} Z^{*} \rightarrow l^{+} l^{-} l^{+} l^{-}$ $(l=e, \mu)$ gives the so-called 'gold-plated' signal for the SM Higgs in the mass range $m_{\varphi}=130-800 \mathrm{GeV}$. Below $m_{\varphi} \sim 130 \mathrm{GeV}$, both the total rate and the acceptance decrease very rapidly, leading to too small a signal for detection. For all three neutral Higgs bosons of the MSSM, the rates in this channnel are always smaller than in the SM. In the case of $A$, there is no $A Z Z$ coupling at tree level, and loop corrections cannot generate measurable 
rates in the four-lepton channel. As for $h$, if $m_{t} \lesssim 180 \mathrm{GeV}$ and $m_{\tilde{q}} \lesssim 1 \mathrm{TeV}$, one can see from fig. 1 that $m_{h} \lesssim 130 \mathrm{GeV}$. Therefore, the $h \rightarrow Z^{*} Z^{*} \rightarrow 4 l$ signal does not have chances of detection at the LHC and SSC, unless one chooses extremely high values for $m_{t}$ and $m_{\tilde{q}}$ or one has superb resolution and acceptance for leptons. The situation is somewhat better in the case of $H$, despite the strong suppression with respect to the SM, due to the competition with the $h h, b \bar{b}, t \bar{t}$ channels, as discussed in sect. 3.

In fig. 25, we show signal rates for the SM Higgs boson and for $H$, for the same choices of parameters as in fig. 15. The threshold effects and the suppression for large values of $\tan \beta$ are clearly visible.

The LHC and SSC discovery potential can be estimated by using the results of simulations carried out for the SM [15,49,50, 16, 18], taking also into account that $\Gamma_{H}<2 \mathrm{GeV}$ all over the mass region of interest, $m_{H} \lesssim 2 m_{t}$. Assuming excellent lepton momentum resolution, in the mass range $2 m_{Z}<$ $m_{H}<2 m_{t}$ a signal rate $\sim 20$ smaller than in the SM could still lead to a detectable signal. In fig. 26 , we show contour plots in the $\left(m_{A}, \tan \beta\right)$ plane, corresponding to fixed values of $\sigma \cdot B R(H \rightarrow 4 l)$. QCD corrections have been included according to ref. [31]. In view of the strong sensitivity to the value of $m_{t}$, we show contours for $m_{t}=120,140,160 \mathrm{GeV}$, for LHC and SSC energies, and for $m_{\tilde{q}}=1 \mathrm{TeV}$. The two almost vertical dashed lines correspond to $m_{H}=2 m_{Z}$ and to $m_{H}=2 m_{t}$. For $m_{H}>2 m_{Z}$, a detectable signal could be obtained up to $\tan \beta \sim 5$. Notice that the experimental acceptances change with $m_{H}$; in particular, in the region $m_{H}<2 m_{Z}$ they fastly decrease with decreasing $m_{H}$ : for a realistic assessment of the discovery limits in this mass region, one should take this and other effects into account. Anyway, the prospects for detection for $m_{H}<2 m_{Z}$ do not look good if $m_{t} \lesssim 150 \mathrm{GeV}$ and $m_{\tilde{q}} \lesssim 1 \mathrm{TeV}$.

The $\tau^{+} \tau^{-}$channel. For the SM Higgs boson, the $\tau^{+} \tau^{-}$decay channel has been found hopelessly difficult for discovery [51,52], since this channel has bad mass resolution and overwhelmingly large background coming from the production of $t \bar{t}, W W+$ jets, Drell-Yan pairs, $Z+$ jets, $b \bar{b}+$ jets, $\ldots$ The bad resolution is due to the fact that the tau-decay products always include one or more neutrinos, which carry away energy; therefore one cannot reconstruct the signal as a resonance peak. The situation is improved if the Higgs is produced with large transverse momentum that is balanced by a jet [53. In 
this case one can use the approximation

$$
p_{\nu}^{(1)} \frac{\vec{p}_{l, T}^{(1)}}{E_{l}^{(1)}}+p_{\nu}^{(2)} \frac{\vec{p}_{l, T}^{(2)}}{E_{l}^{(2)}}=\vec{p}_{\mathrm{T}}^{\text {miss }}
$$

to reconstruct the transverse momenta of the neutrinos and hence the invariant mass of the tau pair. In the above equation, $p_{\nu}^{(i)}$ denotes the total transverse momentum of the neutrinos coming from the decay of $\tau^{(i)}, i=1,2$, while $\vec{p}_{l, T}^{(i)}$ and $E_{l}^{(i)}$ denote the lepton momenta and energies, respectively. It was shown in ref. [51] that, in the mass range $m_{\phi}=70-140 \mathrm{GeV}$, a mass resolution of $\sim 13-17 \%$ can be achieved. This method can also be used for the hadronic decay modes, taking advantage of the fact that the rate is higher by a factor of $\sim 3.5$. When a tau decays hadronically, the hadrons have very low multiplicity and invariant mass, and these properties might be used to recognize the ' $\tau$-jet' [54]. There is a price for the better mass resolution. Tagging on a large- $p_{\mathrm{T}}$ jet can reduce the rate by an order of magnitude. Furthermore, at $10^{5} \mathrm{pb}^{-1}$ /year luminosity, the presence of pile-up deteriorates significantly the measurement of $\vec{p}_{T}^{\text {miss }}$, and therefore the $\tau^{+} \tau^{-}$signal can only be studied with this method at lower luminosities, $\sim 10^{4} \mathrm{pb}^{-1} /$ year . While these difficulties appear prohibitive in the case of the SM, the situation is not entirely negative in the MSSM.

In fig. 27, we display signal rates for $\phi \rightarrow \tau^{+} \tau^{-}$production $(\phi=h, H, A)$, for the same parameter choices as in fig. 15, together with the SM values. We can see that for large values of $\tan \beta$ the production rates can become much larger than in the SM. In the case of $h$ production, for $\tan \beta=30$ the enhancement can be more than one order of magnitude, and increases with decreasing values of $m_{h}$ (see figs. 27a and 27d). Huge enhancements can be obtained also for $H$ and $A$, thanks to the properties of the $\tau^{+} \tau^{-}$ branching ratios discussed in sect. 4 . Note for example that, at the LHC, for $m_{H}, m_{A} \sim 500 \mathrm{GeV}$ and $\tan \beta \gtrsim 10$, we get $\sigma \cdot B R\left(H, A \rightarrow \tau^{+} \tau^{-}\right) \geq 20 \mathrm{pb}$, while for $m_{A}, m_{H} \sim 120 \mathrm{GeV}$ and $\tan \beta \gtrsim 30$, we obtain $\sigma \cdot B R(H, A \rightarrow$ $\left.\tau^{+} \tau^{-}\right) \geq 30 \mathrm{pb}$. The rates for the SSC are rescaled by the factor already discussed in the previous section.

In order to assess, for any given mass, the cross-section values above which one obtains a measurable signal over the large background, detailed

\footnotetext{
${ }^{6}$ Alternatively, at high luminosity one may try to just search for an excess of events in the $e^{ \pm} \mu^{\mp}$ or $l^{ \pm}+$' $\tau$-jet' channels.
} 
simulations are needed. Preliminary studies have been reported for the lepton channel $e^{ \pm} \mu^{\mp}$ in ref. [51] and for the mixed channel $l^{ \pm}+$' $\tau$-jet' in ref. [55]. In the second case, the difficulty of recognizing a ' $\tau$-jet' may be compensated by the higher rate of this channel. The preliminary analysis of ref. 555 finds for the LHC sensitivity to values of $\sigma \cdot B R\left(\phi \rightarrow \tau^{+} \tau^{-}\right)$down to $\sim 10 \mathrm{pb}$ in the low-mass region $m_{\phi} \sim 100 \mathrm{GeV}$ and $\sim 1 \mathrm{pb}$ in the high-mass region $m_{\phi} \sim 400 \mathrm{GeV}$. This result cannot be easily rescaled to the SSC case, since a large mass interval is involved and the SSC luminosity gives more favourable experimental conditions for the srudy of this channel.

In fig. 28, we show contour plots corresponding to fixed values of $\sigma$. $B R\left(\phi \rightarrow \tau^{+} \tau^{-}\right)$, for the same values of $m_{t}$ and $m_{\tilde{q}}$ as in fig. 15 .

\subsection{Charged Higgs boson}

We now move to the discussion of possible physics signals associated with the charged Higgs boson. The phenomenology of the charged Higgs boson at hadron colliders was previously discussed in refs. [56]. The benchmark mass value for charged-Higgs-boson searches at the LHC and SSC is $m_{H^{ \pm}}=$ $m_{t}-m_{b}$. For lower values of $m_{H^{ \pm}}$, the dominant production mechanism at large hadron colliders is $g g \rightarrow t \bar{t}$, followed by $t \rightarrow H^{+} b$. For higher values of $m_{H^{ \pm}}$, the dominant production mechanism is $g b \rightarrow t H^{+}$. As far as detectable signals are concerned, this last case appears hopeless, in view of the suppressed cross-section and of the large backgrounds coming from QCD subprocesses. The first case appears instead to be experimentally viable over a non-negligible region of parameter space. Given the known $t \bar{t}$ production cross-section, one can compute the $t \rightarrow b H^{+}$branching ratio according to well-known formulae, parametrizing again the leading QCD corrections by running masses evaluated at a scale $Q \sim m_{H^{ \pm}}$. The charged Higgs branching ratios were discussed in the previous section, where it was found that the $\tau^{+} \nu_{\tau}$ mode dominates in the mass range under consideration. The experimental signal of a charged Higgs would then be a violation of lepton universality in semileptonic top decays. As a convenient indicator, one can consider the ratio

$$
R=\frac{B R\left(t \rightarrow \tau^{+} \nu_{\tau} b\right)}{B R\left(t \rightarrow \mu^{+} \nu_{\mu} b\right)} \equiv 1+\Delta R
$$


with

$$
\Delta R=\frac{B R\left(t \rightarrow H^{+} b\right) \cdot B R\left(H^{+} \rightarrow \tau^{+} \nu_{\tau}\right)}{B R\left(t \rightarrow W^{+} b\right) \cdot B R\left(W^{+} \rightarrow \mu^{+} \nu_{\mu}\right)} .
$$

Preliminary investigations [56] show that the experimental sensitivity could reach $\Delta R \gtrsim 0.15$ at the LHC. At the SSC the increased $t \bar{t}$ production crosssection is likely to give better sensitivity. In fig. 29, we display contour lines of $\Delta R$ in the $\left(m_{A}, \tan \beta\right)$ plane, for the three representative values $m_{t}=120,140,160 \mathrm{GeV}$. The dashed lines denote the kinematical limit $m_{H^{ \pm}}=m_{t}-m_{b}$. One can see that the most difficult values of $\tan \beta$ are those between 3 and 10, and that the process under consideration could give access to values of $m_{A}$ as high as $80-120 \mathrm{GeV}$ for top-quark masses in the range $120-160 \mathrm{GeV}$.

\section{Conclusions and outlook}

In this paper we carried out a systematic analysis of the possible physics signals of the MSSM Higgs sector at the LHC and SSC, assuming that the supersymmetric (R-odd) particles are heavy enough not to affect significantly the production cross-sections and the branching ratios of the MSSM Higgs particles. As independent parameters in the Higgs sector, we chose $m_{A}$ and $\tan \beta$, and we considered the theoretically motivated region of the parameter space

$$
0 \leq m_{A} \leq 500 \mathrm{GeV}, \quad 1 \leq \tan \beta \leq 50 .
$$

We assumed $m_{\tilde{q}}=1 \mathrm{TeV}$ and negligible mixing in the squark sector. We included the most important radiative corrections to the Higgs masses $m_{h}$, $m_{H}, m_{H^{ \pm}}$, and to the Higgs couplings to fermions and vector bosons. We also included the most important radiative corrections to the three-point couplings of the neutral Higgs bosons.

We estimated the discovery potential of LEP I and LEP II, and we carried out detailed cross-section calculations for the LHC and SSC. We singled out four classes of final states $\left(\gamma \gamma, l^{ \pm} \gamma \gamma, l^{+} l^{-} l^{+} l^{-}, \tau^{+} \tau^{-}\right)$which could provide significant signals for neutral Higgs bosons at the LHC and SSC, and we also examined possible signals of charged Higgs bosons in top decays.

We calculated all the relevant branching ratios, and the cross-sections for all the relevant production mechanisms. We presented our results with the help of branching-ratio curves, cross-section curves, signal-rate curves and 
contour plots in the $\left(m_{A}, \tan \beta\right)$ plane. We did not perform new background studies, but we pointed out that, using the results of our calculations and of the existing simulations carried out for the SM Higgs, supplemented by estimates of the acceptances and efficiencies of typical experiments, in many cases one can draw conclusions concerning the discovery range. In some cases, as for the $\tau^{+} \tau^{-}$channel, further simulation work appears to be needed in order to reach firm conclusions. Nevertheless, some preliminary conclusions can already be drawn and will now be summarized.

At large hadron colliders, the MSSM Higgs search is, in general, more difficult than the SM Higgs search. This is due to the fact that, in a large region of the parameter space, at least one of the MSSM neutral Higgs bosons is in the intermediate mass region, $80 \mathrm{GeV} \lesssim m_{\phi} \lesssim 140 \mathrm{GeV}$, but with rates in the $\gamma \gamma$ and $l \gamma \gamma$ channels which can be significantly suppressed with respect to the SM case. Similarly, neutral Higgs bosons with $m_{\phi} \gtrsim 130 \mathrm{GeV}$ have typically strongly suppressed rates in the $l^{+} l^{-} l^{+} l^{-}$channel. On the contrary, in the MSSM, for rather large values of $\tan \beta$, one can obtain a much larger signal rate in the $\tau^{+} \tau^{-}$channel than in the SM. Finally, $t \rightarrow b H^{+}$decays, followed by $H^{+} \rightarrow \tau^{+} \nu_{\tau}$, can give detectable signals only in a rather restricted region of the parameter space.

As an example, we now try to assess the discovery potential of the different channels for the representative parameter choice $m_{t}=140 \mathrm{GeV}, m_{\tilde{q}}=1 \mathrm{TeV}$, working as usual in the $\left(m_{A}, \tan \beta\right)$ plane.

To begin with, we recall the expectations for LEP II. The size of the LEP II discovery region depends rather strongly on $m_{t}$ and $m_{\tilde{q}}$, and on the assumed energy and luminosity. For standard machine parameters, LEP II cannot test the whole parameter space allowed by the present data. Looking back at fig. 8, one may tentatively say that LEP II will give us (if no Higgs boson is discovered) lower limits of about $m_{A} \gtrsim 70-100 \mathrm{GeV}$ and $\tan \beta \gtrsim$ 3-8 for $m_{t}=120 \mathrm{GeV}, \tan \beta \gtrsim 1.5-3$ for $m_{t}=160 \mathrm{GeV}$.

We observe that the LHC and SSC will test the MSSM Higgs sector in a largely complementary region of the $\left(m_{A}, \tan \beta\right)$ plane. A pictorial summary of the discovery potential of the different channels is presented in fig. 30 . We emphasize once again that the final discovery limits will depend on the machine and detector properties, as well as on the actual values of the top and the soft supersymmetry-breaking masses. We therefore drew fig. 30 just for illustrative purposes, to exemplify a particularly convenient way of considering all the discovery channels at once. 
The $\gamma \gamma$ and $l \gamma \gamma$ channels are important in approximately the same region of the parameter space, $m_{A} \gg m_{Z}$ for $h$ and $50 \mathrm{GeV} \lesssim m_{A} \lesssim 100 \mathrm{GeV}$ for $H$. Therefore, these two channels can be experimentally cross-checked one against the other, reinforcing the significance of a possible signal. As an optimistic discovery limit for $h$, we show in fig. 30 the contour line $\sigma \cdot B R(h \rightarrow$ $\gamma \gamma)=30 \mathrm{fb}$ at the LHC, corresponding to $m_{A} \gtrsim 200 \mathrm{GeV}$. This contour line is shown only for $m_{h} \gtrsim 80 \mathrm{GeV}$. In the region of the parameter space to the right of this line [indicated by the labels $h \rightarrow \gamma \gamma$ and $l+(h \rightarrow \gamma \gamma)$ ], it is expected that measurable signals will be found, assuming detector and machine parameters as discussed in refs. [16]. Approximately the same contour line is obtained by taking $\sigma \cdot B R(h \rightarrow \gamma \gamma)=85 \mathrm{fb}$ at the SSC. This indicates that, in the inclusive $\gamma \gamma$ channel, the discovery range of the LHC and SSC will be the same if the luminosity at the LHC will be $\sim 3$ times higher than at the SSC and if the detectors used at the two machines will have similar efficiencies in suppressing the backgrounds. Very similar discovery lines can be drawn by considering the $l \gamma \gamma$ channel and taking $\sigma \cdot B R[l+(h \rightarrow$ $\gamma \gamma)] \sim 0.8 \mathrm{fb}$ for the LHC and $\sim 4 \mathrm{fb}$ for the SSC.

In fig. 30 we also show the contour line for $\sigma \cdot B R(H \rightarrow \gamma \gamma)=20 \mathrm{fb}$ at the LHC, corresponding to $\sim 55 \mathrm{fb}$ at the SSC. The slightly smaller signal rate was chosen to account for the improved efficiencies at higher Higgs-mass values. The contour line defines a narrow strip around $m_{A} \sim 75 \mathrm{GeV}$ (shaded in fig. 30), where the discovery of $H$ is expected to be possible both in the $\gamma \gamma$ and in the $l \gamma \gamma$ channels [for lack of space the label $l+(H \rightarrow \gamma \gamma)$ has been omitted].

The four-lepton channel is important mainly for $H$, in the mass region $2 m_{Z} \lesssim m_{H} \lesssim 2 m_{t}$, which translates into $150 \mathrm{GeV} \lesssim m_{A} \lesssim 2 m_{t}$, and for relatively small $\tan \beta$. As a reference value for discovery in this mass region, we take $\sigma \cdot B R(H \rightarrow 4 l)=1 \mathrm{fb}$ for the LHC, which corresponds to $\sigma \cdot B R(H \rightarrow 4 l) \sim 3 \mathrm{fb}$ for the SSC. This contour defines the area in fig. 30 indicated by the label $H \rightarrow 4 l$. In a small part of this area, corresponding to $m_{A} \sim m_{t}$ and $\tan \beta \sim 1, A \rightarrow \gamma \gamma$ could also give a detectable signal.

In the region of very large $\tan \beta$, and moderately large $m_{A}$, one could take advantage of the enhanced production cross-sections and of the unsuppressed decays into $\tau^{+} \tau^{-}$to obtain a visible signal for one or more of the MSSM neutral Higgs bosons, and in particular for $H$ and $A$, whose masses can be significantly larger than $100 \mathrm{GeV}$. The simulation work for this process is still at a rather early stage [55], so that no definite conclusion can be drawn 
yet. For reference, the dotted line in fig. 30 corresponds to a (somewhat arbitrary) interpolation of $\sigma \cdot B R\left(\phi \rightarrow \tau^{+} \tau^{-}\right) \sim 10 \mathrm{pb}$ at $m_{\phi}=100 \mathrm{GeV}$ and $\sigma \cdot B R\left(\phi \rightarrow \tau^{+} \tau^{-}\right) \sim 1 \mathrm{pb}$ at $m_{\phi}=400 \mathrm{GeV}$, for LHC energy and summing over the $\phi=H, A$ channels.

Finally, in the region of parameter space corresponding to $m_{A} \lesssim 100 \mathrm{GeV}$, a violation of lepton universality due to the decay chain $t \rightarrow b H^{+} \rightarrow b \tau^{+} \nu_{\tau}$ could indicate the existence of the charged Higgs boson. This region is indicated by the label $H^{ \pm} \rightarrow \tau \nu$ in fig. 30. Its right border is defined by the contour line of $R=1.15$, where $R$ was defined in eq. (42).

By definition, our contour lines do not take into account changes in the acceptances and efficiencies, which are expected in realistic experimental conditions, and depend on the Higgs mass and on the detector. We therefore expect some deformations of our contours once discovery lines are extracted from realistic experimental simulations 43,55.

As a last piece of information we also display in fig. 30 the border of the expected discovery region at LEP II, which depends rather sensitively, as already discussed, on the assumed values of the machine energy and luminosity. We then show two representative lines: the lower dashed line corresponds to $\sigma\left(e^{+} e^{-} \rightarrow h Z, H Z, h A, H A\right)=0.2 \mathrm{pb}$ at $\sqrt{s}=175 \mathrm{GeV}$, while the upper dashed line corresponds to $\sigma\left(e^{+} e^{-} \rightarrow h Z, H Z, h A, H A\right)=0.05 \mathrm{pb}$ at $\sqrt{s}=190 \mathrm{GeV}$.

Using the result summarized in fig. 30, we can draw several important qualitative conclusions:

- The discovery potentials of LEP and the LHC/SSC show a certain complementarity. The discovery region at LEP covers all $\tan \beta$ values at small values of $m_{A}$, and all $m_{A}$ values at small values of $\tan \beta$, while at the LHC/SSC one should be sensitive to the large $\tan \beta$, large $m_{A}$ region.

- One may ask whether the LHC and SSC, combined with LEP II, can explore the full parameter space of the MSSM Higgs sector, being sensitive to at least one signal in each point of the $\left(m_{A}, \tan \beta\right)$ plane, for all plausible values of $m_{t}$ and $m_{\tilde{q}}$. At present, this question cannot be answered positively. The union of the regions where one should find signals at least for one Higgs boson does not cover the whole parameter space: the discovery region has a hole in the middle of the parameter 
space. For our parameter choice, the most difficult region appears to be the cross-hatched area around $m_{A}=150 \mathrm{GeV}$ and $\tan \beta=10$. Therefore we cannot claim yet the existence of a 'no-lose' theorem for the MSSM Higgs search.

- One may also ask if there are regions of parameter space where one can find more than one signal from the MSSM Higgs sector. The answer is that around $m_{A}=200 \mathrm{GeV}$ and $\tan \beta<5$ one can discover $h$ at LEP II and $H$ at the LHC/SSC in the four-lepton channel. There is a somewhat smaller region above $m_{A}=200 \mathrm{GeV}$ where one can also find $h$ in the $\gamma \gamma$ and $l \gamma \gamma$ channels. Furthermore, at high values of $\tan \beta$ $(\gtrsim 20)$ and at $m_{A}>200 \mathrm{GeV}$ one may discover $A$ and $H$ in the $\tau \tau$ channel and $h$ in the $\gamma \gamma$ and $l \gamma \gamma$ channels, although the separation of $H$ and $A$ appears to be impossible, due to their almost perfect degeneracy in mass. This part of the parameter space is inaccessible at LEP II. The discovery region for $H$ in the $\gamma \gamma$ and $l, \gamma \gamma$ channels, corresponding to low values of $m_{A}$, largely overlaps with the LEP II discovery region and with the discovery region related to charged-Higgs production in top decays. In the low $\tan \beta$, for $80 \mathrm{GeV} \lesssim m_{A} \lesssim 180 \mathrm{GeV}$ and $m_{A} \gtrsim 2 m_{t}$ one has a signal at LEP II and no signal at the LHC and SSC, since $m_{h}$ is too small for detection.

Finally, we would like to make some comments on the theoretical uncertainties and on possible future studies.

Our values for the signal rates depend on several phenomenological input parameters, as the value of the bottom mass, the parton-number densities and the value of $\alpha_{S}$. The given cross-sections and branching ratios will change if the input parameters are varied in their allowed range. Also, for some production mechanisms, only the Born cross-sections are known. We estimate that the theoretical errors of the calculated rates vary from about $30 \%$, in the case of the $\gamma \gamma$ channel, up to about a factor of 2 when the $b \bar{b} \phi$ or $t \bar{t} \phi$ production mechanisms are important.

We did not study all effects associated to variations of the parameters in the SUSY $(R$-odd) sector. It would be interesting to examine the case when some of the Higgs bosons are allowed to decay into $R$-odd SUSY particles, or the effects of squark mixing. More importantly, serious simulation work is still needed, in particular for the $\tau^{+} \tau^{-}$and the $l \gamma \gamma$ channel. 


\section{Note added}

After the completion of most of the work presented in this paper, which was anticipated in many talks [57], we received a number of papers [58,59] dealing with different subsets of the material presented here, and reaching similar conclusions. Reference [59] also contains the generalization of eqs. (21 27) to the case of arbitrary mixing in the stop and sbottom mass matrices, but still neglecting the $D$-term contributions.

\section{Acknowledgements}

We are grateful to G. Altarelli for discussions, and for insisting that we should carry out this study. We also thank A. Brignole, D. Denegri, J. Ellis, L. Fayard, D. Froidevaux, J.-F. Grivaz, P. Janot, F. Pauss, G. Ridolfi, C. Seez, T. Sjöstrand, D. Treille, J. Virdee and P. Zerwas for useful discussions and suggestions. 


\section{References}

1. J. Carter, M. Davier and J. Ellis, Rapporteur's talks given at the LPHEP '91 Conference, Geneva, 1991, to appear in the Proceedings, and references therein.

2. M.S. Chanowitz, Ann. Rev. Nucl. Part. Phys. 38 (1988) 323;

M. Sher, Phys. Rep. 179 (1989) 273;

R.N. Cahn, Berkeley preprint LBL-29789 (1990);

G. Altarelli, preprint CERN-TH.6092/91.

3. J.F. Gunion, H.E. Haber, G.L. Kane and S. Dawson, The Higgs Hunter's Guide (Addison-Wesley, 1990).

4. For reviews and references see, e.g.:

H.-P. Nilles, Phys. Rep. 110 (1984) 1;

H.E. Haber and G.L. Kane, Phys. Rep. 117 (1985) 75;

R. Barbieri, Riv. Nuo. Cim. 11 (1988) 1.

5. R. Barbieri, M. Frigeni, F. Giuliani and H.E. Haber, Nucl. Phys. B341 (1990) 309;

A. Bilal, J. Ellis and G.L. Fogli, Phys. Lett. B246 (1990) 459;

M. Drees and K. Hagiwara, Phys. Rev. D42 (1990) 1709;

M. Boulware and D. Finnell, Phys. Rev. D44 (1991) 2054;

M. Drees, K. Hagiwara and A. Yamada, Durham preprint DTP/91/34;

R. Barbieri, M. Frigeni and F. Caravaglios, Pisa preprint IFUP-TH $48 / 91$.

6. G. Altarelli, talk given at the LP-HEP '91 Conference, Geneva, 1991, to appear in the Proceedings, and references therein.

7. G.G. Ross, Rapporteur's talk given at the LP-HEP '91 Conference, Geneva, 1991, to appear in the Proceedings, and references therein.

8. P. Fayet, Nucl. Phys. B90 (1975) 104, Phys. Lett. 64B (1976) 159 and 69B (1977) 489;

R.K. Kaul and P. Majumdar, Nucl. Phys. B199 (1982) 36;

R. Barbieri, S. Ferrara and C.A. Savoy, Phys. Lett. B119 (1982) 36;

H.P. Nilles, M. Srednicki and D. Wyler, Phys. Lett. B120 (1983) 346; 
J.M. Frère, D.R.T. Jones and S. Raby, Nucl. Phys. B222 (1983) 11;

J.-P. Derendinger and C. Savoy, Nucl. Phys. B237 (1984) 307;

J. Ellis, J.F. Gunion, H.E. Haber, L. Roszkowski and F. Zwirner, Phys. Rev. D39 (1989) 844;

U. Ellwanger, preprint HD-THEP-91-21, CERN-TH.6144/91;

P. Binétruy and C.A. Savoy, preprint SPhT/91-143, LPHTE Orsay 91/046;

J.R. Espinosa and M. Quirós, Madrid preprint IEM-FT-50/91.

9. J. Polchinski and L. Susskind, Phys. Rev. D26 (1982) 3661;

J.E. Kim and H.-P. Nilles, Phys. Lett. B138 (1984) 150;

L. Hall, J. Lykken and S. Weinberg, Phys. Rev. D27 (1983) 2359;

G.F. Giudice and A. Masiero, Phys. Lett. 206B (1988) 480;

K. Inoue, M. Kawasaki, M. Yamaguchi and T. Yanagida, Tohoku Univ. preprint TU-373 (1991);

J.E. Kim and H.-P. Nilles, Phys. Lett. B263 (1991) 79;

E.J. Chun, J.E. Kim and H.-P. Nilles, Seoul preprint SNUTP-91-25.

10. H.-P. Nilles, M. Srednicki and D. Wyler, Phys. Lett. B124 (1983) 337; A.B. Lahanas, Phys. Lett. B124 (1983) 341;

L. Alvarez-Gaumé, J. Polchinski and M.B. Wise, Nucl. Phys. B221 (1983) 495;

A. Sen, Phys. Rev. D30 (1984) 2608 and D32 (1985) 411.

11. J.F. Gunion and H.E. Haber, Nucl. Phys. B272 (1986) 1, B278 (1986) 449, and B307 (1988) 445;

J.F. Gunion, G. Gamberini and S.F. Novaes, Phys. Rev. D38 (1988) 3481 ;

T. Weiler and T.C. Yuan, Nucl. Phys. B318 (1989) 337.

12. Z. Kunszt and F. Zwirner, in Proceedings of the Large Hadron Collider Workshop, Aachen, 1990 (G. Jarlskog and D. Rein eds.), Vol. II, p. 578 and Erratum.

13. Y. Okada, M. Yamaguchi and T. Yanagida, Prog. Theor. Phys. Lett. 85 (1991) 1;

J. Ellis, G. Ridolfi and F. Zwirner, Phys. Lett. B257 (1991) 83;

H.E. Haber and R. Hempfling, Phys. Rev. Lett. 66 (1991) 1815. 
14. Z. Kunszt and W.J. Stirling, in Proceedings of the Large Hadron Collider Workshop, Aachen, 1990 (G. Jarlskog and D. Rein eds.), Vol. II, p. 428.

15. D. Froidevaux, in Proceedings of the Large Hadron Collider Workshop, Aachen, 1990 (G. Jarlskog and D. Rein eds.), Vol. II, p. 444.

16. ASCOT, CMS, EAGLE and L3/LHC Collaborations, Expressions of Interest presented at the General Meeting on LHC Physics and Detectors, 5-8 March 1992, Evian-les-Bains, France.

17. R. Craven, ed., Proceedings of the 1990 Summer Study on High Energy Physics, Snowmass, Colo., 1990, to be published, and references therein.

18. Letter of Intent by the Solenoidal Detector Collaboration, November 1990 ;

Letter of Intent by the GEM Collaboration, November 1991.

19. S.P. Li and M. Sher, Phys. Lett. B140 (1984) 339;

J.F. Gunion and A. Turski, Phys. Rev. D39 (1989) 2701, D40 (1989) 2325 and D40 (1989) 2333;

M. Berger, Phys. Rev. D41 (1990) 225.

20. R. Barbieri, M. Frigeni and M. Caravaglios, Phys. Lett. B258 (1991) 167 ;

Y. Okada, M. Yamaguchi and T. Yanagida, Phys. Lett. B262 (1991) 54 ;

A. Yamada, Phys. Lett. B263 (1991) 233;

J.R. Espinosa and M. Quirós, Phys. Lett. B266 (1991) 389;

P.H. Chankowski, S. Pokorski and J. Rosiek, Phys. Lett. B274 (1992) 191 ;

A. Brignole, Padua preprint DFPD/91/TH/28, to appear in Phys. Lett. B;

M. Drees and M.N. Nojiri, preprint KEK-TH-305 (1991);

M.A. Diaz and H.E. Haber, Santa Cruz preprint SCIPP-91-14;

A. Brignole, preprint CERN-TH.6366/92, to appear in Phys. Lett. B; K. Sasaki, M. Carena and C.E.M. Wagner, Munich preprint MPIPh/91-109; 
S. Kelley, J.L. Lopez, D.V. Nanopolous, H. Pois and K. Yuan, Texas preprint CTP-TAMU-104/91.

21. R. Barbieri and M. Frigeni, Phys. Lett. B258 (1991) 395.

22. J. Ellis, G. Ridolfi and F. Zwirner, Phys. Lett. B262 (1991) 477.

23. A. Brignole, J. Ellis, G. Ridolfi and F. Zwirner, Phys. Lett. B271 (1991) 123.

24. A. Brignole, unpublished.

25. D. Decamp et al. (ALEPH Collaboration), Phys. Lett. B265 (1991) 475;

P. Igo-Kemenes (OPAL Collaboration), L. Barone (L3 Collaboration), and W. Ruhlmann (DELPHI Collaboration), talks given at the LPHEP '91 Conference, Geneva, July 1991, to appear in the Proceedings; M. Davier, Rapporteur's talk at the LP-HEP '91 Conference, Geneva, July 1991, to appear in the Proceedings, and references therein.

26. P. Janot, Orsay preprint LAL 91-61 and talk given at the 'Physics at LEP 200' meeting, Paris, February 1992.

27. D. Treille, private communication;

C. Rubbia, Rapporteur's talk given at the LP-HEP '91 Conference, Geneva, 1991, to appear in the Proceedings;

U. Amaldi, plenary talk at the Workshop on Physics and Experiments with Linear Colliders, Saariselkä, September 1991, to appear in the Proceedings.

28. S.G. Gorishny et al., Mod. Phys. Lett. A5 (1990) 2703, and references therein.

29. J. Fleischer and F. Jegerlehner, Phys. Rev. D23 (1981) 2001;

B. Kniehl, Nucl. Phys. B357 (1991) 439;

D.Yu. Bardin, P.Ch. Christova and B.M. Vilenskii, Dubna preprint JINR-P2-91-140.

30. A. Djouadi, M. Spira, J.J. van der Bij and P.M. Zerwas, Phys. Lett. B257 (1991) 187. 
31. A. Djouadi, M. Spira and P.M. Zerwas, Phys. Lett. B264 (1991) 441.

32. A.I. Vainshtein, M.B. Voloshin, V.I. Zakharov amd M.S. Shifman, Sov. J. Nucl. Phys. 30 (1979) 711.

33. J.F. Gunion, G.L. Kane and J. Wudka, Nucl. Phys. B299 (1988) 231;

A. Méndez and A. Pomarol, Nucl. Phys. B349 (1991) 369;

M. Capdequi-Peyranère, H.E. Haber and P. Irulegui, Phys. Rev. D44 (1991) 231.

34. M. Drees and K. Hikasa, Phys. Lett. B240 (1990) 455;

A. Méndez and A. Pomarol, Phys. Lett. B252 (1990) 461;

C.S. Li and R.J. Oakes, Phys. Rev. D43 (1991) 855.

35. P.N. Harriman, A.D. Martin, R.G. Roberts and W.J. Stirling, Phys. Rev. D42 (1990) 798.

36. H.M. Georgi, S.L. Glashow, M.E. Machacek and D.V. Nanopoulos, Phys. Rev. Lett. 40 (1978) 692.

37. M. Chanowitz and M.K. Gaillard, Phys. Lett. 142B (1984) 85 and Nucl. Phys. B261 (1985) 379;

G.L. Kane, W. Repko and W. Rolnick, Phys. Lett. 148B (1984) 367;

R. Cahn and S. Dawson, Phys. Lett. 136B (1984) 196 and (E) 138B (1984) 464.

38. Z. Kunszt, Nucl. Phys. B247 (1984) 339;

V. Barger, A. Stange and R.J.N. Phillips, Phys. Rev. D44 (1991) 1987.

39. R.M. Barnett, E. Haber and D.E. Soper, Nucl. Phys. B306 (1988) 697;

D.A. Dicus and S. Willenbrock, Phys. Rev. D39 (1989) 751.

40. S.L. Glashow, D.V. Nanopoulos and A. Yildiz, Phys. Rev. 18 (1978) 1724 .

41. T. Han and S. Willenbrock, Phys. Lett. B273 (1991) 167.

42. C. Seez et al., in Proceedings of the Large Hadron Collider Workshop, Aachen, 1990 (G. Jarlskog and D. Rein eds.), Vol. II, p. 474.

43. C. Seez and T.S. Virdee, Imperial College preprint IC/HEP/92-4. 
44. L. Fayard and G. Unal, EAGLE Internal Note Physics-NO-001, 1991.

45. R. Kleiss, Z. Kunszt and W.J. Stirling, Phys. Lett. B253 (1991) 269.

46. W. Marciano and F. Paige, Phys. Rev. Lett. 66 (1991) 2433;

J.F. Gunion, Phys. Lett. B262 (1991) 510.

47. Z. Kunszt, Z. Trocsanyi and J.W. Stirling, Phys. Lett. B271 (1991) 247 ;

A. Ballestrero and E. Maina, Phys. Lett. B268 (1991) 437.

48. M. Mangano, SDC Collaboration Note SSC-SDC-90-00113 (1990).

49. A. Nisati, in Proceedings of the Large Hadron Collider Workshop, Aachen, 1990 (G. Jarlskog and D. Rein eds.), Vol. II, p. 494.

50. M. Della Negra et al., in Proceedings of the Large Hadron Collider Workshop, Aachen, 1990 (G. Jarlskog and D. Rein eds.), Vol. II, p. 509.

51. L. Di Lella, in Proceedings of the Large Hadron Collider Workshop, Aachen, 1990 (G. Jarlskog and D. Rein eds.), Vol. II, p. 530.

52. K. Bos, F. Anselmo and B. van Eijk, in Proceedings of the Large Hadron Collider Workshop, Aachen, 1990 (G. Jarlskog and D. Rein eds.), Vol. II, p. 538.

53. R.K. Ellis, I. Hinchliffe, M. Soldate and J.J. van der Bij, Nucl. Phys. B297 (1988) 221.

54. C. Albajar et al. (UA1 Collaboration), Phys. Lett. 185B (1987) 233.

55. F. Pauss, lectures given in the CERN Academic Training Programme, December 1991, and references therein.

56. I. Bigi, Y. Dokshitzer, V. Khoze, J. Kühn and P. Zerwas, Phys. Lett. B181 (1986) 157;

V. Barger and R.J.N. Phillips, Phys. Rev. D41 (1990) 884;

A.C. Bawa, C.S. Kim and A.D. Martin, Z. Phys. C47 (1990) 75;

R.M. Godbole and D.P. Roy, Phys. Rev. D43 (1991) 3640;

M. Felcini, in Proceedings of the Large Hadron Collider Workshop, Aachen, 1990 (G. Jarlskog and D. Rein eds.), Vol. II, p. 414; 
M. Drees and D.P. Roy, Phys. Lett. B269 (1991) 155;

B.K. Bullock, K. Hagiwara and A.D. Martin, Phys. Rev. Lett. 67 (1991) 3055;

D.P. Roy, preprints CERN-TH.6247/91 and CERN-TH.6274/91.

57. Z. Kunszt, talk given to the CMS Collaboration, September 1991; talk given to the L3/LHC Collaboration, September 1991;

F. Zwirner, talk given to the ASCOT Collaboration, September 1991; plenary talk at the Workshop on Physics and Experiments with Linear Colliders, Saariselkä, September 1991 and preprint CERN-TH.6357/91; talk given to the CMS Collaboration, January 1992.

58. J.F. Gunion, R. Bork, H.E. Haber and A.Seiden, Davis preprint UCD91-29, SCIPP-91/34;

H. Baer, M. Bisset, C. Kao and X. Tata, Florida preprint FSU-HEP911104, UH-511-732-91;

J.F. Gunion and L.H. Orr, Davis preprint UCD-91-15;

J.F. Gunion, H.E. Haber and C. Kao, Davis preprint UCD-91-32, SCIPP91/44, FSU-HEP-911222.

59. V. Barger, M.S. Berger, A.L. Stange and R.J.N. Phillips, Univ. Wisconsin preprint MAD-PH-680 (1991) (revised). 


\section{Figure captions}

Fig.1: Contours of $m_{h}^{\max }$ (the maximum value of $m_{h}$, reached for $m_{A} \rightarrow \infty$ ): a) in the $\left(m_{t}, \tan \beta\right)$ plane, for $\left.m_{\tilde{q}}=1 \mathrm{TeV} ; \mathrm{b}\right)$ in the $\left(m_{t}, m_{\tilde{q}}\right)$ plane, for $\tan \beta=m_{t} / m_{b}$.

Fig.2: Contours of $m_{h}$ in the $\left(m_{A}, \tan \beta\right)$ plane, for $m_{\tilde{q}}=1 \mathrm{TeV}$ and a) $m_{t}=$ $120 \mathrm{GeV}$, b) $m_{t}=160 \mathrm{GeV}$.

Fig.3: Contours of $m_{H}$ in the $\left(m_{A}, \tan \beta\right)$ plane, for $m_{\tilde{q}}=1 \mathrm{TeV}$ and a) $m_{t}=$ $120 \mathrm{GeV}, \mathrm{b}) m_{t}=160 \mathrm{GeV}$.

Fig.4: Contours of $m_{H^{ \pm}}$in the $\left(m_{A}, \tan \beta\right)$ plane, for $m_{\tilde{q}}=1 \mathrm{TeV}$. The solid lines correspond to $m_{t}=120 \mathrm{GeV}$, the dashed ones to $m_{t}=160 \mathrm{GeV}$.

Fig.5: Contours of $\sin ^{2} \alpha / \cos ^{2} \beta$ in the $\left(m_{A}, \tan \beta\right)$ plane, for $m_{\tilde{q}}=1 \mathrm{TeV}$. The solid lines correspond to $m_{t}=120 \mathrm{GeV}$, the dashed ones to $m_{t}=$ $160 \mathrm{GeV}$.

Fig.6: Contours of $\cos ^{2} \alpha / \sin ^{2} \beta$ in the $\left(m_{A}, \tan \beta\right)$ plane, for $m_{\tilde{q}}=1 \mathrm{TeV}$. The solid lines correspond to $m_{t}=120 \mathrm{GeV}$, the dashed ones to $m_{t}=$ $160 \mathrm{GeV}$.

Fig.7: Contours of $\sin ^{2}(\beta-\alpha)$ in the $\left(m_{A}, \tan \beta\right)$ plane, for $m_{\tilde{q}}=1 \mathrm{TeV}$. The solid lines correspond to $m_{t}=120 \mathrm{GeV}$, the dashed ones to $m_{t}=$ $160 \mathrm{GeV}$.

Fig.8: Schematic representation of the present LEP I limits and of the future LEP II sensitivity in the $\left(m_{A}, \tan \beta\right)$ plane, for $m_{\tilde{q}}=1 \mathrm{TeV}$ and a) $m_{t}=120 \mathrm{GeV}$, b) $m_{t}=160 \mathrm{GeV}$. The solid lines correspond to the present LEP I limits. The dashed lines correspond to $\sigma\left(e^{+} e^{-} \rightarrow h Z, H Z, h A, H A\right)=0.2 \mathrm{pb}$ at $\sqrt{s}=175 \mathrm{GeV}$, which could be seen as a rather conservative estimate of the LEP II sensitivity. The dash-dotted lines correspond to $\sigma\left(e^{+} e^{-} \rightarrow h Z, H Z, h A, H A\right)=0.05 \mathrm{pb}$ at $\sqrt{s}=190 \mathrm{GeV}$, which could be seen as a rather optimistic estimate of the LEP II sensitivity.

Fig.9: Total widths of the MSSM Higgs bosons, as functions of their respective masses, for $m_{t}=140 \mathrm{GeV}, m_{\tilde{q}}=1 \mathrm{TeV}$ and $\tan \beta=1.5,3,10,30$ : a) $h$; b) $H$; c) $A$; d) $H^{ \pm}$. 
Fig.10: Branching ratios for $h$, as functions of $m_{h}$, for $m_{t}=140 \mathrm{GeV}, m_{\tilde{q}}=$ $1 \mathrm{TeV}$ and: a) $\tan \beta=1.5$; b) $\tan \beta=30$.

Fig.11: Branching ratios for $H$, as functions of $m_{H}$, for $m_{t}=140 \mathrm{GeV}, m_{\tilde{q}}=$ $1 \mathrm{TeV}$ and: a) $\tan \beta=1.5$; b) $\tan \beta=30$.

Fig.12: Branching ratios for $A$, as functions of $m_{A}$, for $m_{t}=140 \mathrm{GeV}, m_{\tilde{q}}=$ $1 \mathrm{TeV}$ and: a) $\tan \beta=1.5$; b) $\tan \beta=30$.

Fig.13: Branching ratios for $h$, as a function of $m_{A}$, for $m_{t}=140 \mathrm{GeV}, m_{\tilde{q}}=$ $1 \mathrm{TeV}$ and: a) $\tan \beta=1.5$; b) $\tan \beta=30$.

Fig.14: Branching ratios for $H^{ \pm}$, as functions of $m_{H^{ \pm}}$, for $m_{t}=140 \mathrm{GeV}$, $m_{\tilde{q}}=1 \mathrm{TeV}$ and: a) $\left.\tan \beta=1.5 ; \mathrm{b}\right) \tan \beta=30$.

Fig.15: Cross-sections for neutral Higgs production, via the gluon-fusion mechanism, as functions of the corresponding masses and for $m_{t}=140 \mathrm{GeV}$, $m_{\tilde{q}}=1 \mathrm{TeV}, \tan \beta=1.5,3,10,30$ : a) $h, \mathrm{LHC}$; b) $H$, LHC; c) $A$, LHC; d) $h, \mathrm{SSC}$; e) $H$, SSC; f) $A$, SSC. QCD corrections are not included.

Fig.16: Cross-sections for $h$ and $H$ production, via the $W$-fusion mechanism, as functions of the corresponding masses, for the same parameter choices as in fig. 15: a) $h$, LHC; b) $H$, LHC; c) $h$, SSC; d) $H$, SSC.

Fig.17: Cross-sections for associated $b \bar{b} \phi$ production, as functions of the corresponding Higgs masses, for the same parameter choices as in fig. 15: a) $h, \mathrm{LHC}$; b) $H$, LHC; c) $A, \mathrm{LHC}$; d) $h$, SSC; e) $H, \mathrm{SSC}$ f) $A$, SSC.

Fig.18: Cross-sections for associated $W \phi$ production, as functions of the corresponding Higgs masses and for the same parameter choices as in fig. 15: a) $h, \mathrm{LHC}$; b) $H$, LHC; c) $h$, SSC; d) $H$, SSC.

Fig.19: Cross-sections for associated $t \bar{t} \phi$ production, as functions of the corresponding Higgs masses and for the same parameter choices as in fig. 15: a) $h, \mathrm{LHC}$; b) $H, \mathrm{LHC}$; c) $A, \mathrm{LHC}$; d) $h$, SSC; e) $H$, SSC; f) $A$, SSC.

Fig.20: Cross-sections times branching ratios for inclusive Higgs production (the gluon-fusion, $W$-fusion, and $b \bar{b} \phi$ contributions are summed) and decay in the $\gamma \gamma$ channel, for the same parameter choices as in fig. 15: a) $h, \mathrm{LHC}$; b) $H$, LHC; c) $A$, LHC; d) $h$, SSC; e) $H$, SSC; f) $A$, SSC. 
For the sake of comparison, the SM values are also indicated. QCD corrections to the gluon-fusion mechanism are not included.

Fig.21: Significance of the inclusive $\phi \rightarrow \gamma \gamma$ signal, in the plane defined by $m_{\phi}$ and $\sigma \cdot B R(\phi \rightarrow \gamma \gamma)$, for the CMS detector proposal at the LHC, with an energy resolution $\Delta E / E=[2 \% / \sqrt{E(\mathrm{GeV})}]+0.5 \%$. The solid lines are contours of constant $S / \sqrt{B}$, where $S$ is the signal and $B$ is the background. The dashed line corresponds to the SM Higgs, including QCD corrections to the gluon-fusion mechanism. Courtesy of C. Seez [43].

Fig.22: Contours of constant cross-sections times branching ratios, in the $\left(m_{A}, \tan \beta\right)$ plane, for the inclusive $\phi \rightarrow \gamma \gamma$ channel: a) $h$, LHC; b) $H$, LHC; c) $h$, SSC; d) $H$, SSC. The choice of $m_{t}$ and $m_{\tilde{q}}$ is the same as in fig. 15 , and QCD corrections to the gluon-fusion mechanism are included.

Fig.23: Cross-sections for associated $W \phi$ and $t \bar{t} \phi$ production, times branching ratios for the $\phi \rightarrow \gamma \gamma$ channel, for the same parameter choices as in fig. 15: a) $W h$, LHC; b) $W H$, LHC; c) $W h$, SSC; d) $W H$, SSC; e) $t \bar{t} h$, LHC; f) $t \bar{t} H$, LHC; g) $t \bar{t} A$, LHC; h) $t \bar{t} h$, SSC; i) $t \bar{t} H$, SSC; j) $t \bar{t} A$, SSC. For the sake of comparison, the SM values are also indicated.

Fig.24: Contours of constant $L_{\phi}=[2 \sigma(t \bar{t} \phi) \cdot B R(t \rightarrow W b)+\sigma(W \phi)] \cdot B R(\phi \rightarrow$ $\gamma \gamma) \cdot B R(W \rightarrow l \nu)$, for the same choice of $m_{t}$ and $m_{\tilde{q}}$ as in fig. 15: a) $h$, LHC; b) $H$, LHC; c) $h$, SSC; d) $H$, SSC.

Fig.25: Cross-sections for inclusive $H$ production (the gluon-fusion, $W$-fusion and $b \bar{b} \phi$ contributions are summed) and decay in the $Z^{*} Z^{*} \rightarrow 4 l^{ \pm}$ channel $(l=e, \mu)$, for the same parameter choices as in fig. 15: a) LHC; b) SSC. For the sake of comparison, the SM values are also indicated. QCD corrections to the gluon-fusion mechanism are not included.

Fig.26: Contours of constant cross-sections times branching ratios for $H \rightarrow$ $Z^{*} Z^{*} \rightarrow 4 l^{ \pm}$, for the same choice of $m_{\tilde{q}}$ as in fig. 15 and: a) $m_{t}=$ $140 \mathrm{GeV}$, LHC; b) $m_{t}=140 \mathrm{GeV}$, SSC; c) $m_{t}=120 \mathrm{GeV}$, LHC; d) $m_{t}=120 \mathrm{GeV}$, SSC; e) $m_{t}=160 \mathrm{GeV}$, LHC; f) $m_{t}=160 \mathrm{GeV}$, SSC. QCD corrections to the gluon-fusion mechanism are included. 
Fig.27: Cross-sections times branching ratios for $\phi \rightarrow \tau^{+} \tau^{-}$, for the same parameter choices as in fig. 15: a) $h$, LHC; b) $H$, LHC; c) $A$, LHC; d) $h$, SSC; e) $H, \mathrm{SSC}$;) $A$, SSC. For the sake of comparison, the SM values are also indicated.

Fig.28: Contours of constant cross-sections times branching ratios for $\phi \rightarrow$ $\tau^{+} \tau^{-}$, for the same choice of $m_{t}$ and $m_{\tilde{q}}$ as in fig. 15: a) $h$, LHC; b) $H$, LHC; c) $A$, LHC; d) $h$, SSC; e) $H$, SSC; f) $A$, SSC. The vertical lines in c) and f) correspond to $m_{A}=60 \mathrm{GeV}$.

Fig.29: Contours of constant $\Delta R=\left[B R\left(t \rightarrow H^{+} b\right) \cdot B R\left(H^{+} \rightarrow \tau^{+} \nu_{\tau}\right)\right] /[B R(t \rightarrow$ $\left.\left.W^{+} b\right) \cdot B R\left(W^{+} \rightarrow \mu^{+} \nu_{\mu}\right)\right]$, for $m_{\tilde{q}}=1 \mathrm{TeV}$ and: a) $m_{t}=140 \mathrm{GeV}$; b) $m_{t}=120 \mathrm{GeV}$; c) $m_{t}=160 \mathrm{GeV}$.

Fig.30: Pictorial summary of the discovery potential of large hadron colliders for $m_{\tilde{q}}=1 \mathrm{TeV}$ and $m_{t}=140 \mathrm{GeV}$. 